\title{
A Viscosity-Splitting Method for the Navier-Stokes/ Darcy Problem
}

\author{
Yunxia Wang ${ }^{1,2}$, Xuefeng $\operatorname{Han}^{3}$ and Zhiyong $\mathrm{Si}^{4, *}$ \\ 1 School of Materials Science and Engineering, Henan Polytechnic University, Jiaozuo, \\ Henan 454003, China \\ ${ }^{2}$ Henan Joint International Research Laboratory for High Performance Metallic \\ Material and Their Numerical Simulation, Henan Polytechnic University, Jiaozuo, \\ Henan 454003, China \\ ${ }^{3}$ School of Mathematics and Information Science, Henan Polytechnic University, Jiaozuo, \\ Henan 454003, China \\ 4 School of Mathematics and Computational Science, Xiangtan University, Xiangtan, \\ Hunan 411105, China
}

Received 29 March 2019; Accepted (in revised version) 28 May 2019

\begin{abstract}
In this report, we give a viscosity splitting method for the NavierStokes/Darcy problem. In this method, the Navier-Stokes/Darcy equation is solved in three steps. In the first step, an explicit/ implicit formulation is used to solve the nonlinear problem. We introduce an artificial diffusion term $\theta \Delta \mathbf{u}$ in our scheme whose purpose is to enlarge the time stepping and enhance numerical stability, especially for small viscosity parameter $v$, by choosing suitable parameter $\theta$. In the second step, we solve the Stokes equation for velocity and pressure. In the third step, we solve the Darcy equation for the piezometric head in the porous media domain. We use the numerical solutions at last time level to give the interface condition to decouple the Navier-Stokes equation and the Darcy's equation. The stability analysis, under some condition $\Delta t \leq k_{0}, k_{0}>0$, is given. The error estimates prove our method has an optimal convergence rates. Finally, some numerical results are presented to show the performance of our algorithm.
\end{abstract}

AMS subject classifications: 76D05, 35Q30, 65M60, 65N30

Key words: Navier-Stokes/Darcy equations, fractional step method, viscosity-splitting method, stability analysis, optimal error analysis.

${ }^{*}$ Corresponding author.

Emails: wangyunxia@hpu.edu.cn (Y. X. Wang), hanxuefeng@hpu.edu.cn (X. F. Han), sizhiyong@hpu.edu.cn (Z. Y. Si) 


\section{Introduction}

The free flow coupling with the porous media flow, where the behavior of the fluid can be described by different partial differential equations in different domains, is very important in the computational fluids. In this report, we focus on the model of the NavierStokes equation in the surface region coupling the Darcy's law in the subsurface region [16], which is described by a mixed Navier-Stokes/Darcy's model. The first important thing is the interface conditions between the Navier-Stokes flow and the Darcy's flow. This model was firstly studied by Beavers and Joseph in [4], they gave the interface condition (named Beavers-Joseph (BJ) condition) on the interface between the surface flow and the subsurface flow. Then, this condition was simplified by Saffman [37] getting the Beavers-Joseph-Saffman (BJS) condition. This model is used in many areas, e.g., the simulation of flooding in dry areas, petroleum engineering and environmental engineering. In point view of the numerical method, how to deal with the BJS or BJ condition on the interface is a enormous challenge.

For its widely used and enormous challenge, many authors have given some great works, e.g., unified stabilized finite element formulations for the Stokes/Darcy's flow [2]; a strongly conservative finite element method for the Stokes/Darcy's flow [30]; superconvergence analysis of finite element method for the Stokes/Darcy system [1]; a posteriori error estimate for the Stokes/Darcy [15] and so on. In 2012, Layton et al. [31] split the Stokes/Darcy problems into the Stokes and Darcy problems and give four noniterative, sub-physics, uncoupling methods. The Robin-Robin domain decomposition methods for the steady-state Stokes-Darcy system with the Beavers-Joseph interface condition were shown by Cao et al. [9], Chen et al. [12] and Discacciati et al. [17]. In [23], fully-mixed finite element methods was given by Gatica et al. In [36], a decoupled finite element method for the Stokes/Darcy flow was given. In 2012, Shan, Zheng and Layton [38] presented a decoupled method using different time steps in different domains. In [41], a local discontinuous Galerkin (LDG) method for the Stokes/Darcy flow was given by Vassilev and Yotov. For the Navier-Stokes/Darcy coupling problem, several iterative methods were presented by Badea, Discacciatiaw and Quarteroni $[3,16]$. The two-level method for the Navier-Stokes/Darcy problem was given by Cai et al. [7] in 2009. Girault and Riviére [25] presented a discontinuous Galerkin approximation of coupled Navier-Stokes/Darcy Equations by BJS Interface Condition. In [43], two decoupling algorithms for the steady Stokes-Darcy model based on two-grid discretizations were shown by Zhang and Yuan. In [39], we gave the decoupled modified characteristics finite element method for the time dependent Navier-Stokes/Darcy problem. The fullymixed finite element method for Stokes-Darcy problems was given by Gatica et al. [8,24]. A decoupled preconditioning technique for a mixed Stokes-Darcy model was presented by Márquez et al. [32]. A strong coupling of finite element methods for the Stokes-Darcy problem was shown by Márquez et al. [33]. Two-grid finite element for mixed StokesDarcy equations was given by Hou et al. $[29,44]$.

The fractional step methods, which split effects due to different operators appeared in 
the problem, are a widely used classical numerical method for time-dependent differential equations in computational fluids. The origin of this method is given by Chorin [11] and Temam [40]. They developed the important and well known projection method. The main drawbacks of projection methods are that the end-of-step velocity does not satisfy the exact boundary conditions and the discrete pressure should satisfy a so-called 'artificial' boundary conditions. The viscosity-splitting methods is a kind of the fraction step method, where viscosity is not fully decoupled from incompressibility. The fully discrete viscosity-splitting method was called $\theta$-scheme given by Glowinski et al. [27] and FernáCara et al. [20]. In [5,6], Blasco et al. gave a viscosity-splitting fractional step method for the Navier-Stokes equation and a new error estimate for which was give by F. GuillénGonzález and Redondo-Neble [26]. The fractional step method and operator-splitting scheme combined with the well-known predictor-multi-corrector algorithm for solving the Navier-Stokes problem were also considered in $[13,14,18,19]$. The advancement of this method is decomposed into a sequence of two steps. At the first step, a linear elliptic problem was solved, while at the second step a Stokes problem was considered. In [42], a large time stepping viscosity-splitting fractional-step method was considered for the viscoelastic flow problem.

In this paper, we given a viscosity splitting method for the Navier-Stokes/Darcy problem. In the first step, the explicit/implicit formulation is used to solve the nonlinear problem. We use the implicit scheme for the linear terms and an explicit scheme for the nonlinear term. The advantage of this method is that a linear system with a constant coefficient matrix can be obtained in order to save computational cost. Furthermore, we introduce an artificial diffusion term $\theta \Delta \mathbf{u}$ in our scheme for solving the small viscosity $v$. The purpose of the artificial diffusion term $\theta \Delta \mathbf{u}$ is to enlarge the time stepping and enhance numerical stability especially for the small viscosity parameter by choosing suitable parameter $\theta$. In the second step, a Stokes equation is solved. At last, we solve Darcy problems in the porous media domain. Then, we give the numerical analysis including stability analysis and error analysis. The numerical result show that our method has an optimal convergence order. On the other hand, we can see that our method can solve the Navier-Stokes/Darcy problem with small viscosity parameter $v$. The numerical analysis proves that our method is stable and has an optimal convergence rate. The numerical results conform our theoretical analysis.

\section{The viscosity splitting method for the time dependent Navier-Stokes/Darcy problems and functional settings}

Let $\Omega \subset \mathbb{R}^{d}(d=2,3)$ be a bounded domain, decomposed into two non intersecting subdomains $\Omega_{f}$ and $\Omega_{p}$ separated by an interface $\Gamma$, namely $\Omega=\Omega_{f} \cup \Omega_{p}, \Omega_{f} \cap \Omega_{p}=\varnothing$ and $\bar{\Omega}_{f} \cap \bar{\Omega}_{p}=\Gamma$. We suppose the boundaries $\partial \Omega_{f}$ and $\partial \Omega_{p}$ have the Lipschitz conditions. From the physical point of view, $\Gamma$ is a surface separating the domain $\Omega_{f}$ filled by a fluid from a domain $\Omega_{p}$ form by a porous medium. 
Let $T>0$ be a finite constant, the fluid flow is governed by the Navier-Stokes equation in $\Omega_{f}$

$$
\begin{cases}\mathbf{u}_{t}-v \Delta \mathbf{u}+(\mathbf{u} \cdot \nabla) \mathbf{u}+\nabla p=f(x, t), & x \in \Omega_{f} \times(0, T] \\ \nabla \cdot \mathbf{u}=0, & x \in \Omega_{f} \times(0, T] \\ \mathbf{u}(x, 0)=\mathbf{u}_{0}, & x \in \Omega_{f}, \\ \mathbf{u}=0, & x \in \partial \Omega_{f} \backslash \Gamma \times(0, T]\end{cases}
$$

where $\mathbf{u}(x, t)$ represents the velocity of the fluid flow in $\Omega_{f}, p(x, t)$ is the pressure, $f(x, t)$ is the external body force and $v$ is the kinematic viscosity.

The porous media flow is governed by the following equation in $\Omega_{p}$

$$
\begin{cases}S_{0} \phi_{t}+\nabla \cdot \mathbf{u}_{p}=g_{p}(x, t), & x \in \Omega_{p} \times(0, T] \\ \mathbf{u}_{p}=-\mathbf{K} \nabla \phi, & x \in \Omega_{p} \times(0, T] \\ \phi(x, 0)=\phi_{0}, & x \in \Omega_{p}, \\ \phi=0, & x \in \partial \Omega_{p} \backslash \Gamma \times(0, T],\end{cases}
$$

where $\phi$ is the piezometric head, $\mathbf{u}_{p}$ is the fluid velocity in the porous media $\Omega_{p}$. K represents the hydraulic conductivity tensor, for simplicity, we assume that $\mathbf{K}=\operatorname{diag}$, $\left(K_{1}, \cdots, K_{d}\right)$ with $K_{i} \in L^{\infty}\left(\Omega_{p}\right), i=1, \cdots, d$ and $K_{i}>0, i=1, \cdots, d$, which means the porous media is homogeneous. We assume that $\mathbf{K}$ is uniformly bounded and positive defined in $\Omega_{p}$, there exist $K_{\min }, K_{\max }>0$ such that

$$
K_{\min }|x|^{2} \leq \mathbf{K} x \cdot x \leq K_{\max }|x|^{2} \quad \text { a.e. } x \in \Omega_{p} .
$$

Using Darcy's law $\mathbf{u}_{p}=-\mathbf{K} \nabla \phi$, (2.2) can be rewritten in the parabolic form

$$
S_{0} \phi_{t}-\nabla \cdot(\mathbf{K} \nabla \phi)=g_{p}(x, t), \quad x \in \Omega_{p} \times(0, T] .
$$

For the Navier-Stokes/Darcy model, the interface conditions of the conservation of mass, balance of forces and the Beavers-Joseph-Saffman condition are imposed herein

$$
\begin{array}{ll}
\mathbf{u} \cdot \mathbf{n}_{f}+\mathbf{u}_{p} \cdot \mathbf{n}_{p}=0 & \text { on } \Gamma \times(0, T], \\
p-v \mathbf{n}_{f} \frac{\partial \mathbf{u}}{\partial \mathbf{n}_{f}}=g \phi & \text { on } \Gamma \times(0, T], \\
-v \tau_{i} \frac{\partial \mathbf{u}}{\partial \mathbf{n}_{f}}=\frac{\alpha \sqrt{v g}}{\sqrt{\operatorname{trace}(\mathbf{K})}} \mathbf{u} \cdot \tau_{i}, \quad i=1,2, \cdots, d-1, & \text { on } \Gamma \times(0, T] .
\end{array}
$$

Here, $g$ is the gravitational acceleration, $\alpha$ is a positive parameter depending on the properties of the porous medium and must be determined experimentally. The condition (2.4) can be rewritten as

$$
\mathbf{u} \cdot \mathbf{n}_{f}=\mathbf{K} \frac{\partial \phi}{\partial \mathbf{n}_{p}} \quad \text { on } \quad \Gamma \times(0, T]
$$




\subsection{Variational formulation of the continuous problem}

Define $W=H_{f} \times H_{p}$ and $Q=L^{2}\left(\Omega_{f}\right)$, where

$$
\begin{aligned}
& H_{f}=\left\{\mathbf{v} \in\left(H^{1}\left(\Omega_{f}\right)\right)^{d}: \mathbf{v}=0 \text { on } \partial \Omega_{f} \backslash \Gamma\right\}, \\
& H_{p}=\left\{\phi \in H^{1}\left(\Omega_{p}\right): \phi=0 \text { on } \partial \Omega_{p} \backslash \Gamma\right\} .
\end{aligned}
$$

The spaces $H_{f}$ and $H_{p}$ are equipped with the following norms

$$
\begin{aligned}
& \|\mathbf{u}\|_{H_{f}}=\|\nabla \mathbf{u}\|_{\Omega_{f}}, \quad \forall \mathbf{u} \in H_{f}, \\
& \|\phi\|_{H_{p}}=\|\nabla \phi\|_{\Omega_{p}}, \quad \forall \phi \in H_{p} \text {, }
\end{aligned}
$$

where $\|\cdot\|_{D}:=\|\cdot\|_{L^{2}(D)}$ means the $L^{2}$-norm on the domain $D$. We equip the space $W$ with the following norms

$$
\begin{array}{ll}
\|w\|_{0}=\sqrt{(\mathbf{u}, \mathbf{u})_{\Omega_{f}}+g S_{0}(\phi, \phi)_{\Omega_{p}}}, & \forall w=(\mathbf{u}, \phi) \in W, \\
\|w\|_{W}=\sqrt{v(\nabla \mathbf{u}, \nabla \mathbf{u})_{\Omega_{f}}+g(\mathbf{K} \nabla \phi, \nabla \phi)_{\Omega_{p}}}, & \forall w=(\mathbf{u}, \phi) \in W,
\end{array}
$$

where $(\cdot, \cdot)_{D}$ refers the inner product in the corresponding domain $D$ for $D=\Omega_{f}$ or $\Omega_{p}$. The weak formulation of the time-dependent Navier-Stokes/Darcy model read as follows: find $w=(\mathbf{u}, \phi) \in W$ and $p \in Q$ such that

$$
\begin{array}{ll}
{\left[w_{t}, z\right]+a(w, z)+B(\mathbf{u}, \mathbf{u}, \mathbf{v})+b(\mathbf{v}, p)=(F, z),} & \forall z=(\mathbf{v}, \psi) \in W, \\
b(\mathbf{u}, q)=0, & \forall q \in Q, \\
w(0)=w_{0}, &
\end{array}
$$

where

$$
\begin{array}{ll}
{\left[w_{t}, z\right]=\left(\mathbf{u}_{t}, \mathbf{v}\right)_{\Omega_{f}}+g S_{0}\left(\phi_{t}, \psi\right)_{\Omega_{p},}} & a(w, z)=a_{f}(\mathbf{u}, \mathbf{v})+a_{p}(\phi, \psi)+a_{\Gamma}(w, z), \\
a_{f}(\mathbf{u}, \mathbf{v})=v(\nabla \mathbf{u}, \nabla \mathbf{v})_{\Omega_{f}}+\sum_{i=1}^{d-1} \int_{\Gamma} \frac{\alpha \sqrt{v g}}{\sqrt{\operatorname{trace}(\mathbf{K})}}\left(\mathbf{u} \cdot \tau_{i}\right)\left(\mathbf{v} \cdot \tau_{i}\right) d s, \\
a_{p}(\phi, \psi)=g(\mathbf{K} \nabla \phi, \nabla \psi)_{\Omega_{p},} & a_{\Gamma}(w, z)=a_{\Gamma}(\mathbf{u}, \phi ; \mathbf{v}, \psi)=c_{\Gamma}(\phi, \mathbf{v})-c_{\Gamma}(\psi, \mathbf{u}), \\
B(\mathbf{u}, \mathbf{v}, \mathbf{w})=((\mathbf{u} \cdot \nabla) \mathbf{v}, \mathbf{w}), & c_{\Gamma}(\psi, \mathbf{u})=g \int_{\Gamma} \psi \mathbf{u} \cdot \mathbf{n}_{f}, \\
b(\mathbf{v}, p)=-(p, \nabla \cdot \mathbf{v})_{\Omega_{p}}, & (F, z)=(f, \mathbf{v})_{\Omega_{f}}+g\left(g_{p}, \psi\right)_{\Omega_{p}} .
\end{array}
$$

Here, we define the discrete Stokes operator $\mathcal{A}$ (see [42] and the references there) defined by

$$
(\mathcal{A} \mathbf{u}, \mathbf{v})=\left(\mathcal{A}^{1 / 2} \mathbf{u}, \mathcal{A}^{1 / 2} \mathbf{v}\right)=(\nabla \mathbf{u}, \nabla \mathbf{v}), \quad \forall \mathbf{u}, \mathbf{v} \in H_{f}
$$


Lemma 2.1 ([34]). The bilinear form $a_{f}(\cdot, \cdot), a_{p}(\cdot, \cdot)$ satisfy

$$
\begin{aligned}
& a_{f}(\mathbf{u}, \mathbf{v}) \leq \max \left\{v+1, \frac{C \alpha}{2 \sqrt{K_{\min }}}\right\}\|\mathbf{u}\|_{H_{f}}\|\mathbf{v}\|_{H_{f}}, \\
& a_{f}(\mathbf{u}, \mathbf{u}) \geq v\|\mathbf{u}\|_{H_{f}}^{2}+\frac{\alpha \sqrt{v g}}{\sqrt{K_{\max }}} \sum_{i=1}^{d} \int_{\Gamma}\left(\mathbf{u} \cdot \tau_{i}\right)^{2} d s=: v\|\mathbf{u}\|_{H_{f}}^{2}+\frac{\alpha \sqrt{v g}}{\sqrt{K_{\max }}}\|\mathbf{u} \cdot \tau\|_{\Gamma}^{2}, \\
& a_{p}(\phi, \psi) \leq K_{\max }\|\phi\|_{H_{p}}\|\psi\|_{H_{p}} \\
& a_{p}(\phi, \phi) \geq K_{\min }\|\phi\|_{H_{p}}^{2} .
\end{aligned}
$$

The trilinear form $B(\cdot, \cdot, \cdot)$ satisfies the following property (see [21])

$$
|B(\mathbf{u}, \mathbf{v}, \mathbf{w})| \leq N\|\mathbf{u}\|_{H_{f}}\|\mathbf{v}\|_{H_{f}}\|\mathbf{w}\|_{H_{f}}, \quad \forall \mathbf{u}, \mathbf{v}, \mathbf{w} \in H_{f}
$$

We can get the following lemma

Lemma 2.2. The trilinear form $B(\cdot, \cdot, \cdot)$ satisfies the following property

$$
|B(\mathbf{u}, \mathbf{v}, \mathbf{w})| \leq C\|\mathbf{u}\|_{H^{2}\left(\Omega_{f}\right)}\|\nabla \mathbf{v}\|_{\Omega_{f}}\|\mathbf{w}\|_{\Omega_{f}}, \quad \forall \mathbf{u} \in H^{2}\left(\Omega_{f}\right), \quad \mathbf{v}, \mathbf{w} \in H^{1}\left(\Omega_{f}\right) .
$$

Proof. By Cauchy-Schwarz inequality, we have

$$
|B(\mathbf{u}, \mathbf{v}, \mathbf{w})| \leq\|\mathbf{u}\|_{L^{\infty}\left(\Omega_{f}\right)}\|\nabla \mathbf{v}\|_{\Omega_{f}}\|\mathbf{w}\|_{\Omega_{f}} .
$$

Using the embedding theorem, there holds $\|\mathbf{u}\|_{L^{\infty}\left(\Omega_{f}\right)} \leq C\|\mathbf{u}\|_{H^{2}\left(\Omega_{f}\right)}$, then it follows that

$$
|B(\mathbf{u}, \mathbf{v}, \mathbf{w})| \leq C\|\mathbf{u}\|_{H^{2}\left(\Omega_{f}\right)}\|\nabla \mathbf{v}\|_{\Omega_{f}}\|\mathbf{w}\|_{\Omega_{f}} .
$$

Thus, we complete the proof.

Remark 2.1. Here, for $\mathbf{u} \notin H_{0}^{1}\left(\Omega_{f}\right) \cap H^{2}\left(\Omega_{f}\right)$, so we can not get

$$
\|\mathbf{u}\|_{H^{2}\left(\Omega_{f}\right)} \leq C\|\mathcal{A} \mathbf{u}\|_{\Omega_{f}} .
$$

\subsection{The Viscosity-Splitting for the Navier-Stokes/Darcy problems}

For each positive integer $N$, let $\left\{\mathcal{J}_{n}: 1 \leq n \leq N\right\}$ be a partition of $[0, T]$ into subintervals $\mathcal{J}_{n}=\left(t_{n-1}, t_{n}\right]$, with $t_{n}=n \Delta t, \Delta t=T / N$. With the previous notations, we get the viscosity splitting method for the Navier-Stokes/Darcy problem (2.1).

Algorithm 1 Viscosity-Splitting method.

Step 1 Find $\mathbf{u}^{n+1 / 2} \in H_{f}$, such that

$$
\frac{\mathbf{u}^{n+1 / 2}-\mathbf{u}^{n}}{\Delta t}-v \Delta \mathbf{u}^{n+1 / 2}+\theta \mathcal{A} \mathbf{u}^{n+1 / 2}-\theta \mathcal{A} \mathbf{u}^{n}+\left(\mathbf{u}^{n} \cdot \nabla\right) \mathbf{u}^{n}=f\left(t_{n+1}\right) .
$$


Step 2 Find $\left(\mathbf{u}^{n+1}, p^{n+1}\right) \in\left(H_{f}, Q\right)$, such that

$$
\begin{aligned}
& \frac{\mathbf{u}^{n+1}-\mathbf{u}^{n+1 / 2}}{\Delta t}-v\left(\Delta \mathbf{u}^{n+1}-\Delta \mathbf{u}^{n+1 / 2}\right)+\theta\left(\mathcal{A} \mathbf{u}^{n+1}-\mathcal{A} \mathbf{u}^{n+1 / 2}\right)+\nabla p^{n+1}=0, \\
& \nabla \cdot \mathbf{u}^{n+1}=0 .
\end{aligned}
$$

Step 3 Find $\phi^{n+1} \in H_{p}$, via

$$
S_{0} \frac{\phi^{n+1}-\phi^{n}}{\Delta t}+\nabla \cdot\left(\mathbf{K} \nabla \phi^{n}\right)=g_{p}\left(x, t_{n+1}\right)
$$

Then, we can get the weak form of the method

Algorithm 2 Weak form of the Viscosity-Splitting method.

Step 1 Find $\mathbf{u}^{n+1 / 2} \in H_{f}$, such that

$$
\begin{aligned}
& \left(\frac{\mathbf{u}^{n+1 / 2}-\mathbf{u}^{n}}{\Delta t}, \mathbf{v}\right)_{\Omega_{f}}+a_{f}\left(\mathbf{u}^{n+1 / 2}, \mathbf{v}\right)+\theta\left(\nabla \mathbf{u}^{n+1 / 2}, \nabla \mathbf{v}\right) \Omega_{f}-\theta\left(\nabla \mathbf{u}^{n}, \nabla \mathbf{v}\right) \Omega_{f} \\
& +B\left(\mathbf{u}^{n}, \mathbf{u}^{n}, \mathbf{v}\right)+c_{\Gamma}\left(\phi^{n}, \mathbf{v}\right)=\left(f\left(t_{n+1}\right), \mathbf{v}\right)_{\Omega_{f}} .
\end{aligned}
$$

Step 2 Find $\left(\mathbf{u}^{n+1}, p^{n+1}\right) \in\left(H_{f}, Q\right)$, such that

$$
\begin{aligned}
& \left(\frac{\mathbf{u}^{n+1}-\mathbf{u}^{n+1 / 2}}{\Delta t}, \nabla \mathbf{v}\right)_{\Omega_{f}}+a_{f}\left(\mathbf{u}^{n+1}-\mathbf{u}^{n+1 / 2}, \mathbf{v}\right) \\
& \quad+\theta\left(\nabla \mathbf{u}^{n+1}-\nabla \mathbf{u}^{n+1 / 2}, \nabla \mathbf{v}\right)_{\Omega_{f}}+b\left(\mathbf{v}, p^{n+1}\right)=0, \\
& b\left(\mathbf{u}^{n+1}, q\right)=0 .
\end{aligned}
$$

Step 3 Find $\phi^{n+1} \in H_{p}$, via

$$
\begin{gathered}
g S_{0}\left(\frac{\phi^{n+1}-\phi^{n}}{\Delta t}, \psi\right)_{\Omega_{p}}+g\left(\mathbf{K} \nabla \phi^{n+1}, \nabla \psi\right)_{\Omega_{p}} \\
-c_{\Gamma}\left(\psi, \mathbf{u}^{n}\right)=g\left(g_{p}\left(x, t_{n+1}\right), \psi\right)_{\Omega_{p}} .
\end{gathered}
$$

\section{Stability analysis}

Lemma $3.1([31,36])$. There exits a constant $C>0$, such that

$$
\left|c_{\Gamma}(\phi, \mathbf{u})\right| \leq C g\|\phi\|_{H_{p}}\|\mathbf{u}\|_{H_{f}} .
$$


Theorem 3.1 (Stability). The Viscosity-Splitting method is stable, in the sense that, under the condition $k_{0}>0, \Delta t<k_{0}$, there hold

$$
\begin{aligned}
& \left\|\mathbf{u}^{n+1}\right\|_{\Omega_{f}}^{2}+\sum_{i=0}^{n}\left\|\mathbf{u}^{i+1}-\mathbf{u}^{i+1 / 2}\right\|_{\Omega_{f}}^{2}+\frac{\Delta t v}{2} \sum_{i=0}^{n}\left\|\mathbf{u}^{i+1 / 2}\right\|_{H_{f}}^{2} \\
& +\frac{\alpha \sqrt{v g}}{\sqrt{K_{\max }}} \sum_{i=0}^{n}\left(\left\|\mathbf{u}^{i+1} \cdot \tau\right\|_{\Gamma}^{2}+\left\|\mathbf{u}^{i+1} \cdot \tau-\mathbf{u}^{i+1 / 2} \cdot \tau\right\|_{\Gamma}^{2}\right)+\theta \Delta t\left(\left\|\mathbf{u}^{n+1}\right\|_{H_{f}}\right. \\
& \left.+\sum_{i=0}^{n}\left\|\mathbf{u}^{i+1 / 2}-\mathbf{u}^{i}\right\|_{H_{f}}^{2}+\sum_{i=0}^{n}\left\|\mathbf{u}^{i+1}-\mathbf{u}^{i+1 / 2}\right\|_{H_{f}}^{2}\right)+\Delta t v \sum_{i=0}^{n}\left(\left\|\mathbf{u}^{i+1}\right\|_{H_{f}}^{2}+\left\|\mathbf{u}^{i+1}-\mathbf{u}^{i+1 / 2}\right\|_{H_{f}}^{2}\right) \\
& \quad+g S_{0}\left(\left\|\phi^{n+1}\right\|_{\Omega_{p}}^{2}+\sum_{i=0}^{n}\left\|\phi^{i+1}-\phi^{i}\right\|_{\Omega_{p}}^{2}\right)+2 g \Delta t \sum_{i=0}^{n}\left\|\mathbf{K}^{1 / 2} \nabla \phi^{i+1}\right\|_{\Omega_{p}}^{2} \\
& \leq \exp \left(\frac{4 T C_{2}^{2} v^{-1} g^{2}}{K_{\min }}+\frac{4 C_{2}^{2} g T}{K_{\min }}\right)\left(4 \Delta t v^{-1} \sum_{i=0}^{n}\left\|f\left(t_{n+1}\right)\right\|_{\Omega_{f}}^{2}+\frac{4 \Delta t g}{K_{\min }} \sum_{i=0}^{n}\left\|g_{p}\left(t_{i+1}\right)\right\|_{\Omega_{f}}^{2}\right) \\
& \left\|\mathbf{u}^{n+1}\right\|_{H_{f}}^{2}+2 S_{0}\left\|\phi^{n+1}\right\|_{H_{p}}^{2}+\sum_{i=1}^{n}\left(\left\|\mathbf{u}^{i+1}-\mathbf{u}^{i}\right\|_{H_{f}}^{2}+2 S_{0}\left\|\phi^{i+1}-\phi^{i}\right\|_{H_{p}}^{2}\right) \\
& \quad+\frac{3}{2} \Delta t \sum_{i=1}^{n}\left(v\left\|\mathcal{A} \mathbf{u}^{i+1}\right\|_{\Omega_{f}}^{2}+K_{\min }\left\|\mathcal{A} \phi^{i+1}\right\|_{\Omega_{p}}^{2}\right)+2 \theta \Delta t\left\|\mathcal{A} \mathbf{u}^{n+1}\right\|_{\Omega_{f}}^{2}+2 \theta \Delta t \sum_{i=1}^{n}\left\|\mathcal{A}\left(\mathbf{u}^{i+1}-\mathbf{u}^{i}\right)\right\|_{\Omega_{f}}^{2} \\
& \leq \exp \left(4 g \Delta t v^{-1} \sum_{i=1}^{n}\left\|\mathbf{u}^{i}\right\|_{H^{2}\left(\Omega_{f}\right)}^{2}+4 v^{-1} T+\frac{4 T}{K_{\min }}\right) \\
& \quad \times\left(4 \Delta t v^{-1} \sum_{i=1}^{n}\left\|f\left(t_{i+1}\right)\right\|_{\Omega_{f}}^{2}+\frac{4 \Delta t}{K_{\min }} \sum_{i=0}^{n}\left\|g_{p}\left(t_{i+1}\right)\right\|_{\Omega_{p}}^{2}\right) .
\end{aligned}
$$

Furthermore, we have

$$
(v+\theta) \Delta t \sum_{i=0}^{n}\left\|\mathcal{A} \mathbf{u}^{i+1}\right\|_{\Omega_{f}} \leq C
$$

Proof. Here, we use mathematical induction. It is obviously that

$$
(v+\theta)\left\|\mathcal{A} \mathbf{u}^{0}\right\|_{\Omega_{f}} \leq C .
$$

We assume that

$$
(v+\theta)\left\|\mathcal{A} \mathbf{u}^{i}\right\|_{\Omega_{f}} \leq C, \quad \forall i \leq n .
$$

Step 1. we give the proof of (3.2a) under the induction assumption.

Then, letting $\mathbf{v}=2 \Delta t \mathbf{u}^{n+1 / 2}$ in (2.13), it follows by

$$
\begin{aligned}
& \left\|\mathbf{u}^{n+1 / 2}\right\|_{\Omega_{f}}^{2}-\left\|\mathbf{u}^{n}\right\|_{\Omega_{f}}^{2}+\left\|\mathbf{u}^{n+1 / 2}-\mathbf{u}^{n}\right\|_{\Omega_{f}}^{2}+2 \Delta t v\left\|\mathbf{u}^{n+1 / 2}\right\|_{H_{f}}^{2}+\frac{\alpha \Delta t \sqrt{v g}}{\sqrt{K_{\max }}}\left\|\mathbf{u}^{n+1 / 2} \cdot \tau\right\|_{\Gamma}^{2} \\
& \quad+\theta \Delta t\left(\left\|\mathbf{u}^{n+1 / 2}\right\|_{H_{f}}-\left\|\mathbf{u}^{n}\right\|_{H_{f}}^{2}+\left\|\mathbf{u}^{n+1 / 2}-\mathbf{u}^{n}\right\|_{H_{f}}^{2}\right) \\
& \quad+2 \Delta t B\left(\mathbf{u}^{n}, \mathbf{u}^{n}, \mathbf{u}^{n+1 / 2}\right)+2 \Delta t c_{\Gamma}\left(\phi^{n}, \mathbf{u}^{n+1 / 2}\right)=2 \Delta t\left(f\left(t_{n+1}\right), \mathbf{u}^{n+1 / 2}\right)_{\Omega_{f}} .
\end{aligned}
$$


Combining (2.8) and (2.9) gives

$$
\begin{aligned}
& 2 \Delta t B\left(\mathbf{u}^{n}, \mathbf{u}^{n}, \mathbf{u}^{n+1 / 2}\right) \leq C \Delta t\left\|\mathbf{u}^{n}\right\|_{H^{2}\left(\Omega_{f}\right)}\left\|\mathbf{u}^{n+1 / 2}\right\|_{H_{f}}\left\|\mathbf{u}^{n}\right\|_{\Omega_{f}} \\
\leq & \frac{v \Delta t}{4}\left\|\mathbf{u}^{n+1 / 2}\right\|_{H_{f}}^{2}+C \Delta t\left\|\mathbf{u}^{n}\right\|_{H^{2}\left(\Omega_{f}\right)}^{2}\left\|\mathbf{u}^{n}\right\|_{\Omega_{f}}^{2} .
\end{aligned}
$$

By (3.1), we arrive at

$$
\begin{aligned}
& 2 \Delta t c_{\Gamma}\left(\phi^{n}, \mathbf{u}^{n+1 / 2}\right) \leq 2 \Delta t C_{2} g\left\|\phi^{n}\right\|_{H_{p}}\left\|\mathbf{u}^{n+1 / 2}\right\|_{H_{f}} \\
\leq & 4 \Delta t C_{2}^{2} v^{-1} g^{2}\left\|\phi^{n}\right\|_{H_{p}}^{2}+\frac{v \Delta t}{4}\left\|\mathbf{u}^{n+1 / 2}\right\|_{H_{f}}^{2} \\
\leq & \frac{4 \Delta t C_{2}^{2} v^{-1} g^{2}}{K_{\min }}\left\|\mathbf{K}^{1 / 2} \nabla \phi^{n}\right\|_{\Omega_{p}}^{2}+\frac{v \Delta t}{4}\left\|\mathbf{u}^{n+1 / 2}\right\|_{H_{f}}^{2} .
\end{aligned}
$$

Using the Cauchy-Schwarz inequality, we have

$$
2 \Delta t\left(f\left(t_{n+1}\right), \mathbf{u}^{n+1 / 2}\right)_{\Omega_{f}} \leq 4 C \Delta t v^{-1}\left\|f\left(t_{n+1}\right)\right\|_{\Omega_{f}}^{2}+\frac{v \Delta t}{4}\left\|\mathbf{u}^{n+1 / 2}\right\|_{H_{f}}^{2} .
$$

Combining (3.4)-(3.7), we arrive at

$$
\begin{aligned}
& \left\|\mathbf{u}^{n+1 / 2}\right\|_{\Omega_{f}}^{2}-\left\|\mathbf{u}^{n}\right\|_{\Omega_{f}}^{2}+\left\|\mathbf{u}^{n+1 / 2}-\mathbf{u}^{n}\right\|_{\Omega_{f}}^{2}+\Delta t v\left\|\mathbf{u}^{n+1 / 2}\right\|_{H_{f}}^{2}+\frac{\alpha \Delta t \sqrt{v g}}{\sqrt{K_{\max }}}\left\|\mathbf{u}^{n+1 / 2} \cdot \tau\right\|_{\Gamma}^{2} \\
& \quad+\theta \Delta t\left(\left\|\mathbf{u}^{n+1 / 2}\right\|_{H_{f}}-\left\|\mathbf{u}^{n}\right\|_{H_{f}}^{2}+\left\|\mathbf{u}^{n+1 / 2}-\mathbf{u}^{n}\right\|_{H_{f}}^{2}\right) \\
& \leq 4 \Delta t v^{-1} C_{1}^{2}\left\|\mathbf{u}^{n}\right\|_{H^{2}\left(\Omega_{f}\right.}^{2}\left\|\mathbf{u}^{n}\right\|_{\Omega_{f}}^{2}+\frac{4 \Delta t C_{2}^{2} v^{-1} g^{2}}{K_{\min }}\left\|\mathbf{K}^{1 / 2} \nabla \phi^{n}\right\|_{\Omega_{p}}^{2}+4 C \Delta t v^{-1}\left\|f\left(t_{n+1}\right)\right\|_{\Omega_{f}}^{2} .
\end{aligned}
$$

Taking $\mathbf{v}=2 \Delta t \mathbf{u}^{n+1}$ in (2.14) and noting $b\left(\mathbf{u}^{n+1}, q\right)=0$, we deduce

$$
\begin{aligned}
& \left\|\mathbf{u}^{n+1}\right\|_{\Omega_{f}}^{2}-\left\|\mathbf{u}^{n+1 / 2}\right\|_{\Omega_{f}}^{2}+\left\|\mathbf{u}^{n+1}-\mathbf{u}^{n+1 / 2}\right\|_{\Omega_{f}} \\
& \quad+\Delta t(\theta+v)\left(\left\|\mathbf{u}^{n+1}\right\|_{H_{f}}^{2}-\left\|\mathbf{u}^{n+1 / 2}\right\|_{H_{f}}^{2}+\left\|\mathbf{u}^{n+1}-\mathbf{u}^{n+1 / 2}\right\|_{H_{f}}^{2}\right) \\
& \quad+\frac{\Delta t \alpha \sqrt{v g}}{\sqrt{K_{\max }}}\left(\left\|\mathbf{u}^{n+1} \cdot \tau\right\|_{\Gamma}^{2}-\left\|\mathbf{u}^{n+1 / 2} \cdot \tau\right\|_{\Gamma}^{2}+\left\|\mathbf{u}^{n+1} \cdot \tau-\mathbf{u}^{n+1 / 2} \cdot \tau\right\|_{\Gamma}^{2}\right) \leq 0 .
\end{aligned}
$$

Combining it with (3.8), it follows by

$$
\begin{gathered}
\left\|\mathbf{u}^{n+1}\right\|_{\Omega_{f}}^{2}-\left\|\mathbf{u}^{n}\right\|_{\Omega_{f}}^{2}+\left\|\mathbf{u}^{n+1}-\mathbf{u}^{n+1 / 2}\right\|_{\Omega_{f}}^{2}+\Delta t v\left\|\mathbf{u}^{n+1 / 2}\right\|_{H_{f}}^{2}+\left\|\mathbf{u}^{n+1 / 2}-\mathbf{u}^{n}\right\|_{\Omega_{f}}^{2} \\
+\frac{\Delta t \alpha \sqrt{v g}}{\sqrt{K_{\max }}}\left(\left\|\mathbf{u}^{n+1} \cdot \tau\right\|_{\Gamma}^{2}+\left\|\mathbf{u}^{n+1} \cdot \tau-\mathbf{u}^{n+1 / 2} \cdot \tau\right\|_{\Gamma}^{2}\right)+\theta \Delta t\left(\left\|\mathbf{u}^{n+1}\right\|_{H_{f}}-\left\|\mathbf{u}^{n}\right\|_{H_{f}}^{2}\right. \\
\left.+\left\|\mathbf{u}^{n+1 / 2}-\mathbf{u}^{n}\right\|_{H_{f}}^{2}+\left\|\mathbf{u}^{n+1}-\mathbf{u}^{1 / 2}\right\|_{H_{f}}^{2}\right)+\Delta t v\left(\left\|\mathbf{u}^{n+1}\right\|_{H_{f}}^{2}+\left\|\mathbf{u}^{n+1}-\mathbf{u}^{n+1 / 2}\right\|_{H_{f}}^{2}\right) \\
\leq 4 \Delta t v^{-1} C_{1}^{2}\left\|\mathbf{u}^{n}\right\|_{H^{2}\left(\Omega_{f}\right)}^{2}\left\|\mathbf{u}^{n}\right\|_{\Omega_{f}}^{2}+\frac{4 \Delta t C_{2}^{2} v^{-1} g^{2}}{K_{\min }}\left\|\mathbf{K}^{1 / 2} \nabla \phi^{n}\right\|_{\Omega_{p}}^{2}+4 \Delta t v^{-1}\left\|f\left(t_{n+1}\right)\right\|_{\Omega_{f}}^{2} .
\end{gathered}
$$


Letting $\psi=2 \Delta t \phi^{n+1}$ in (2.15), there holds

$$
\begin{aligned}
& 2 g S_{0}\left(\phi^{n+1}-\phi^{n}, \phi^{n+1}\right)_{\Omega_{p}}+2 g \Delta t\left(\mathbf{K} \nabla \phi^{n+1}, \nabla \phi^{n+1}\right)_{\Omega_{p}}-2 \Delta t c_{\Gamma}\left(\phi^{n+1}, \mathbf{u}^{n}\right) \\
= & 2 g \Delta t\left(g_{p}\left(t_{n+1}\right), \phi^{n+1}\right)_{\Omega_{p}} .
\end{aligned}
$$

Using (3.1), we deduce

$$
\begin{aligned}
& 2 \Delta t c_{\Gamma}\left(\phi^{n+1}, \mathbf{u}^{n}\right) \leq \frac{2 \Delta t C_{2} g}{\sqrt{K_{\min }}}\left\|\mathbf{K}^{1 / 2} \nabla \phi^{n+1}\right\|_{\Omega_{p}}\left\|\mathbf{u}^{n}\right\|_{H_{f}} \\
\leq & \frac{g \Delta t}{4}\left\|\mathbf{K}^{1 / 2} \nabla \phi^{n+1}\right\|_{\Omega_{p}}^{2}+\frac{4 C_{2}^{2} g \Delta t}{K_{\min }}\left\|\mathbf{u}^{n}\right\|_{H_{f}}^{2} .
\end{aligned}
$$

Using the Cauchy-Schwarz inequality, it shows that

$$
2 g \Delta t\left(g_{p}\left(t_{n+1}\right), \phi^{n+1}\right)_{\Omega_{p}} \leq \frac{4 \Delta t g}{K_{\min }}\left\|g_{p}\left(t_{n+1}\right)\right\|_{\Omega_{p}}^{2}+\frac{g \Delta t}{4}\left\|\mathbf{K}^{1 / 2} \nabla \phi^{n+1}\right\|_{\Omega_{p}}^{2} .
$$

Combining (3.9), (3.10) and (3.11) gives

$$
\begin{aligned}
& g S_{0}\left(\left\|\phi^{n+1}\right\|_{\Omega_{p}}^{2}-\left\|\phi^{n}\right\|_{\Omega_{p}}^{2}+\left\|\phi^{n+1}-\phi^{n}\right\|_{\Omega_{p}}^{2}\right)+g \Delta t\left\|\mathbf{K}^{1 / 2} \nabla \phi^{n+1}\right\|_{\Omega_{p}}^{2} \\
\leq & \frac{4 \Delta t g}{K_{\min }}\left\|g_{p}\left(t_{n+1}\right)\right\|_{\Omega_{p}}^{2}+\frac{4 C_{2}^{2} g \Delta t}{K_{\min }}\left\|\mathbf{u}^{n}\right\|_{H_{f}}^{2} .
\end{aligned}
$$

Combining (3.9) and (3.12), we arrive at

$$
\begin{aligned}
& \left\|\mathbf{u}^{n+1}\right\|_{\Omega_{f}}^{2}-\left\|\mathbf{u}^{n}\right\|_{\Omega_{f}}^{2}+\left\|\mathbf{u}^{n+1}-\mathbf{u}^{n+1 / 2}\right\|_{\Omega_{f}}^{2}+\frac{\Delta t v}{2}\left\|\mathbf{u}^{n+1 / 2}\right\|_{H_{f}}^{2} \\
& +\theta \Delta t\left(\left\|\mathbf{u}^{n+1}\right\|_{H_{f}}-\left\|\mathbf{u}^{n}\right\|_{H_{f}}^{2}+\left\|\mathbf{u}^{n+1 / 2}-\mathbf{u}^{n}\right\|_{H_{f}}^{2}\right)+\Delta t v\left(\left\|\mathbf{u}^{n+1}\right\|_{H_{f}}^{2}+\left\|\mathbf{u}^{n+1}-\mathbf{u}^{n+1 / 2}\right\|_{H_{f}}^{2}\right) \\
& \quad+\frac{\Delta t \alpha \sqrt{v g}}{\sqrt{K_{\max }}}\left(\left\|\mathbf{u}^{n+1} \cdot \tau\right\|_{\Gamma}^{2}+\left\|\mathbf{u}^{n+1} \cdot \tau-\mathbf{u}^{n+1 / 2} \cdot \tau\right\|_{\Gamma}^{2}\right) \\
& \quad+g S_{0}\left(\left\|\phi^{n+1}\right\|_{\Omega_{p}}^{2}-\left\|\phi^{n}\right\|_{\Omega_{p}}^{2}+\left\|\phi^{n+1}-\phi^{n}\right\|_{\Omega_{p}}^{2}\right)+2 g \Delta t\left\|\mathbf{K}^{1 / 2} \nabla \phi^{n+1}\right\|_{\Omega_{p}}^{2} \\
& \leq C_{1}^{2} \Delta t v^{-1}\left\|\mathbf{u}^{n}\right\|_{H^{2}\left(\Omega_{f}\right)}^{2}\left\|\mathbf{u}^{n}\right\|_{\Omega_{f}}^{2}+\frac{4 \Delta t C_{2}^{2} v^{-1} g^{2}}{K_{\min }}\left\|\mathbf{K}^{1 / 2} \phi^{n}\right\|_{H_{p}}^{2}+4 \Delta t v^{-1}\left\|f\left(t_{n+1}\right)\right\|_{\Omega_{f}}^{2} \\
& \quad+\frac{4 \Delta t g}{K_{\min }}\left\|g_{p}\left(t_{n+1}\right)\right\|_{\Omega_{p}}^{2}+\frac{4 C_{2}^{2} g t}{K_{\min }}\left\|\mathbf{u}^{n}\right\|_{H_{f}}^{2} .
\end{aligned}
$$

Summing (3.13) over all $n$, there holds

$$
\begin{aligned}
& \left\|\mathbf{u}^{n+1}\right\|_{\Omega_{f}}^{2}+\sum_{i=0}^{n}\left\|\mathbf{u}^{i+1}-\mathbf{u}^{i+1 / 2}\right\|_{\Omega_{f}}^{2}+\frac{\Delta t v}{2} \sum_{i=0}^{n}\left\|\mathbf{u}^{i+1 / 2}\right\|_{H_{f}}^{2} \\
& +\frac{\alpha \sqrt{v g}}{\sqrt{K_{\max }}} \sum_{i=0}^{n}\left(\left\|\mathbf{u}^{i+1} \cdot \tau\right\|_{\Gamma}^{2}+\left\|\mathbf{u}^{i+1} \cdot \tau-\mathbf{u}^{i+1 / 2} \cdot \tau\right\|_{\Gamma}^{2}\right)+\theta \Delta t\left(\left\|\mathbf{u}^{n+1}\right\|_{H_{f}}\right. \\
& \left.+\sum_{i=0}^{n}\left\|\mathbf{u}^{i+1 / 2}-\mathbf{u}^{i}\right\|_{H_{f}}^{2}+\sum_{i=0}^{n}\left\|\mathbf{u}^{i+1}-\mathbf{u}^{i+1 / 2}\right\|_{H_{f}}^{2}\right)+\Delta t v \sum_{i=0}^{n}\left(\left\|\mathbf{u}^{i+1}\right\|_{H_{f}}^{2}+\left\|\mathbf{u}^{i+1}-\mathbf{u}^{i+1 / 2}\right\|_{H_{f}}^{2}\right)
\end{aligned}
$$




$$
\begin{aligned}
& +g S_{0}\left(\left\|\phi^{n+1}\right\|_{\Omega_{p}}^{2}+\sum_{i=0}^{n}\left\|\phi^{i+1}-\phi^{i}\right\|_{\Omega_{p}}^{2}\right)+2 g \Delta t \sum_{i=0}^{n}\left\|\mathbf{K}^{1 / 2} \nabla \phi^{i+1}\right\|_{\Omega_{p}}^{2} \\
& \leq C_{1}^{2} \Delta t v^{-1} \sum_{i=0}^{n}\left\|\mathcal{A} \mathbf{u}^{i}\right\|_{\Omega_{f}}^{2}\left\|\mathbf{u}^{i}\right\|_{\Omega_{f}}^{2}+\frac{4 \Delta t C_{2}^{2} v^{-1} g^{2}}{K_{\min }} \sum_{i=0}^{n}\left\|\mathbf{K}^{1 / 2} \nabla \phi^{i}\right\|_{\Omega_{p}}^{2}+4 \Delta t v^{-1} \sum_{i=0}^{n}\left\|f\left(t_{i+1}\right)\right\|_{\Omega_{f}}^{2} \\
& +\frac{4 \Delta t g}{K_{\min }}\left\|g_{p}\left(t_{i+1}\right)\right\|_{\Omega_{p}}^{2}+\frac{4 C_{2}^{2} g \Delta t}{K_{\min }} \sum_{i=0}^{n}\left\|\mathbf{u}^{i}\right\|_{H_{f}}^{2} .
\end{aligned}
$$

Via Gronwall's lemma three times, it yields

$$
\begin{aligned}
& \left\|\mathbf{u}^{n+1}\right\|_{\Omega_{f}}^{2}+\sum_{i=0}^{n}\left\|\mathbf{u}^{i+1}-\mathbf{u}^{i+1 / 2}\right\|_{\Omega_{f}}^{2}+\frac{\Delta t v}{2} \sum_{i=0}^{n}\left\|\mathbf{u}^{i+1 / 2}\right\|_{H_{f}}^{2} \\
& +\frac{\alpha \sqrt{\nu g}}{\sqrt{K_{\max }}} \sum_{i=0}^{n}\left(\left\|\mathbf{u}^{i+1} \cdot \tau\right\|_{\Gamma}^{2}+\left\|\mathbf{u}^{i+1} \cdot \tau-\mathbf{u}^{i+1 / 2} \cdot \tau\right\|_{\Gamma}^{2}\right)+\theta \Delta t\left(\left\|\mathbf{u}^{n+1}\right\|_{H_{f}}\right. \\
& \left.+\sum_{i=0}^{n}\left\|\mathbf{u}^{i+1 / 2}-\mathbf{u}^{i}\right\|_{H_{f}}^{2}+\sum_{i=0}^{n}\left\|\mathbf{u}^{i+1}-\mathbf{u}^{i+1 / 2}\right\|_{H_{f}}^{2}\right)+\Delta t v \sum_{i=0}^{n}\left(\left\|\mathbf{u}^{i+1}\right\|_{H_{f}}^{2}+\left\|\mathbf{u}^{i+1}-\mathbf{u}^{i+1 / 2}\right\|_{H_{f}}^{2}\right) \\
& +g S_{0}\left(\left\|\phi^{n+1}\right\|_{\Omega_{p}}^{2}+\sum_{i=0}^{n}\left\|\phi^{i+1}-\phi^{i}\right\|_{\Omega_{p}}^{2}\right)+2 g \Delta t \sum_{i=0}^{n}\left\|\mathbf{K}^{1 / 2} \nabla \phi^{i+1}\right\|_{\Omega_{p}}^{2} \\
& \leq \exp \left(\frac{C_{1}^{2} \Delta t}{v} \sum_{i=0}^{n}\left\|\mathcal{A} \mathbf{u}^{i}\right\|_{\Omega_{f}}^{2}+\frac{4 T C_{2}^{2} v^{-1} g^{2}}{K_{\min }}+\frac{4 C_{2}^{2} g T}{K_{\min }}\right) \\
& \quad \times\left(4 \Delta t v^{-1} \sum_{i=0}^{n}\left\|f\left(t_{n+1}\right)\right\|_{\Omega_{f}}^{2}+\frac{4 \Delta t g}{K_{\min }} \sum_{i=0}^{n}\left\|g_{p}\left(t_{i+1}\right)\right\|_{\Omega_{p}}^{2}\right) .
\end{aligned}
$$

Step 2: we give the proof of (3.2b) under the induction assumption.

Adding (2.13) and (2.14), it follows by

$$
\begin{aligned}
& \left(\frac{\mathbf{u}^{n+1}-\mathbf{u}^{n}}{\Delta t}, \mathbf{v}\right)_{\Omega_{f}}+a_{f}\left(\mathbf{u}^{n+1}, \mathbf{v}\right)_{\Omega_{f}}+\theta\left(\nabla\left(\mathbf{u}^{n+1}-\mathbf{u}^{n}\right), \nabla \mathbf{v}\right)_{\Omega_{f}}+B\left(\mathbf{u}^{n}, \mathbf{u}^{n}, \mathbf{v}\right) \\
& +b\left(\mathbf{v}, p^{n+1}\right)+c_{\Gamma}\left(\phi^{n}, \mathbf{v}\right)=\left(f\left(t_{n+1}\right), \mathbf{v}\right)_{\Omega_{f}} .
\end{aligned}
$$

Letting $\mathbf{v}=2 \Delta t \mathcal{A} \mathbf{u}^{n+1}$, we deduce

$$
\begin{aligned}
& 2\left(\mathbf{u}^{n+1}-\mathbf{u}^{n}, \mathcal{A} \mathbf{u}^{n+1}\right)_{\Omega_{f}}+2 \Delta t v\left\|\mathcal{A} \mathbf{u}^{n+1}\right\|_{\Omega_{f}}^{2} \\
& \quad+2 \theta \Delta t\left(\left\|\mathcal{A} \mathbf{u}^{n+1}\right\|_{\Omega_{f}}^{2}-\left\|\mathcal{A} \mathbf{u}^{n}\right\|_{\Omega_{f}}^{2}+\left\|\mathcal{A}\left(\mathbf{u}^{n+1}-\mathbf{u}^{n}\right)\right\|_{\Omega_{f}}^{2}\right) \\
& \quad+2 \Delta t B\left(\mathbf{u}^{n}, \mathbf{u}^{n}, \mathcal{A} \mathbf{u}^{n+1}\right)+2 \Delta t c_{\Gamma}\left(\phi^{n}, \mathcal{A} \mathbf{u}^{n+1}\right) \\
& \leq 2 \Delta t\left(f\left(t_{n+1}\right), \mathcal{A} \mathbf{u}^{n+1}\right)_{\Omega_{f}} .
\end{aligned}
$$


Using the property of $B(\cdot, \cdot, \cdot)$, we have

$$
\begin{aligned}
& 2 \Delta t B\left(\mathbf{u}^{n}, \mathbf{u}^{n}, \mathcal{A} \mathbf{u}^{n+1}\right) \leq 2 C \Delta t\left\|\mathbf{u}^{n}\right\|_{H^{2}\left(\Omega_{f}\right)}\left\|\mathbf{u}^{n}\right\|_{H_{f}}\left\|\mathcal{A} \mathbf{u}^{n+1}\right\|_{\Omega_{f}} \\
\leq & \frac{\Delta t v}{4}\left\|\mathcal{A} \mathbf{u}^{n+1}\right\|_{\Omega_{f}}^{2}+4 C v^{-1} \Delta t\left\|\mathbf{u}^{n}\right\|_{H^{2}\left(\Omega_{f}\right)}^{2}\left\|\mathbf{u}^{n}\right\|_{H_{f}}^{2} .
\end{aligned}
$$

On the other hand, there holds that

$$
\begin{aligned}
& 2 \Delta t c_{\Gamma}\left(\phi^{n}, \mathcal{A} \mathbf{u}^{n+1}\right)=g \int_{\Gamma} \phi^{n} \mathcal{A} \mathbf{u}^{n+1} \cdot n d s=-2 \Delta \operatorname{tg} \int_{\Gamma} \nabla \phi^{n} \cdot \nabla \mathbf{u}^{n+1} \cdot n d s \\
\leq & 2 \Delta t g\left\|\nabla \phi^{n}\right\|_{L^{2}(\Gamma)}\left\|\nabla \mathbf{u}^{n+1}\right\|_{L^{2}(\Gamma)} \leq 2 \Delta \operatorname{tg}\left\|\mathcal{A} \phi^{n}\right\|_{\Omega_{f}}\left\|\mathcal{A} \mathbf{u}^{n+1}\right\|_{\Omega_{f}} \\
\leq & \frac{\Delta t v}{4}\left\|\mathcal{A} \mathbf{u}^{n+1}\right\|_{\Omega_{f}}^{2}+4 g \Delta t v^{-1}\left\|\mathcal{A} \phi^{n}\right\|_{\Omega_{p}}^{2} .
\end{aligned}
$$

Via Cauchy-Schwarz inequality, we get

$$
2 \Delta t\left(f\left(t_{n+1}\right), \mathcal{A} \mathbf{u}^{n+1}\right)_{\Omega_{f}} \leq \frac{\Delta t v}{4}\left\|\mathcal{A} \mathbf{u}^{n+1}\right\|_{\Omega_{f}}^{2}+4 \Delta t v^{-1}\left\|f\left(t_{n+1}\right)\right\|_{\Omega_{f}}^{2} .
$$

Combining these inequalities, it shows that

$$
\begin{aligned}
& \left\|\mathbf{u}^{n+1}\right\|_{H_{f}}^{2}-\left\|\mathbf{u}^{n}\right\|_{H_{f}}^{2}+\left\|\mathbf{u}^{n+1}-\mathbf{u}^{n}\right\|_{H_{f}}^{2}+2 \Delta t v\left\|\mathcal{A} \mathbf{u}^{n+1}\right\|_{\Omega_{f}}^{2} \\
& \quad+2 \theta \Delta t\left(\left\|\mathcal{A} \mathbf{u}^{n+1}\right\|_{\Omega_{f}}^{2}-\left\|\mathcal{A} \mathbf{u}^{n}\right\|_{\Omega_{f}}^{2}+\left\|\mathcal{A}\left(\mathbf{u}^{n+1}-\mathbf{u}^{n}\right)\right\|_{\Omega_{f}}^{2}\right) \\
& \leq \Delta t v\left\|\mathcal{A} \mathbf{u}^{n+1}\right\|_{\Omega_{f}}^{2}+4 \Delta t v^{-1}\left\|f\left(t_{n+1}\right)\right\|_{\Omega_{f}}^{2}+4 g \Delta t v^{-1}\left\|\mathcal{A} \mathbf{K}^{1 / 2} \phi^{n}\right\|_{\Omega_{p}}^{2} \\
& \quad+4 v^{-1} \Delta t\left\|\mathbf{u}^{n}\right\|_{H^{2}\left(\Omega_{f}\right)}^{2}\left\|\mathbf{u}^{n}\right\|_{H_{f}}^{2} .
\end{aligned}
$$

Summing the above inequality all over $n$, we arrive at

$$
\begin{aligned}
& \left\|\mathbf{u}^{n+1}\right\|_{H_{f}}^{2}+\sum_{i=1}^{n}\left\|\mathbf{u}^{i+1}-\mathbf{u}^{i}\right\|_{H_{f}}^{2}+\frac{3}{2} \Delta t v \sum_{i=1}^{n}\left\|\mathcal{A} \mathbf{u}^{i+1}\right\|_{\Omega_{f}}^{2} \\
& \quad+2 \theta \Delta t\left\|\mathcal{A} \mathbf{u}^{n+1}\right\|_{\Omega_{f}}^{2}+2 \theta \Delta t \sum_{i=1}^{n}\left\|\mathcal{A}\left(\mathbf{u}^{i+1}-\mathbf{u}^{i}\right)\right\|_{\Omega_{f}}^{2} \\
& \leq 4 \Delta t v^{-1} \sum_{i=1}^{n}\left\|f\left(t_{i+1}\right)\right\|_{\Omega_{f}}^{2}+4 g \Delta t v^{-1} \sum_{i=1}^{n}\left\|\mathcal{A} \phi^{i}\right\|_{\Omega_{p}}^{2}+4 v^{-1} \Delta t \sum_{i=1}^{n}\left\|\mathbf{u}^{i}\right\|_{H^{2}\left(\Omega_{f}\right)}^{2}\left\|\mathbf{u}^{i}\right\|_{H_{f}}^{2} .
\end{aligned}
$$

Letting $\psi=2 \Delta t \mathcal{A} \phi^{n+1}$ in (2.15), we have

$$
\begin{aligned}
& 2 S_{0}\left(\phi^{n+1}-\phi^{n}, \mathcal{A} \phi^{n+1}\right)_{\Omega_{p}}+2 \Delta t\left(\nabla \mathbf{K} \nabla \phi^{n+1}, \mathcal{A} \phi^{n+1}\right) \\
= & 2 \Delta t c_{\Gamma}\left(\mathcal{A} \phi^{n+1}, \mathbf{u}^{n}\right)+2 \Delta t\left(g_{p}\left(x, t_{n+1}\right), \mathcal{A} \phi^{n+1}\right)_{\Omega_{p}} .
\end{aligned}
$$

Using the definition of $c_{\Gamma}(\cdot, \cdot)$, there holds that

$$
2 \Delta t c_{\Gamma}\left(\mathcal{A} \phi^{n+1}, \mathbf{u}^{n}\right) \leq \frac{K_{\min } \Delta t}{4}\left\|\mathcal{A} \phi^{n+1}\right\|_{\Omega_{p}}^{2}+\frac{4 \Delta t}{K_{\min }}\left\|\mathcal{A} \mathbf{u}^{n}\right\|_{\Omega_{f}}^{2} .
$$


Using Cauchy-Schwarz inequality, we get

$$
2 \Delta t\left(g_{p}\left(x, t_{n+1}\right), \mathcal{A} \phi^{n+1}\right)_{\Omega_{p}} \leq \frac{K_{\min } \Delta t}{4}\left\|\mathcal{A} \phi^{n+1}\right\|_{\Omega_{p}}^{2}+\frac{4 \Delta t}{K_{\min }}\left\|g_{p}\left(t_{n+1}\right)\right\|_{\Omega_{p}}^{2} .
$$

Combining these three inequalities gives

$$
\begin{aligned}
& 2 S_{0}\left(\left\|\phi^{n+1}\right\|_{H_{p}}^{2}-\left\|\phi^{n}\right\|_{H_{p}}^{2}+\left\|\phi^{n+1}-\phi^{n}\right\|_{H_{p}}^{2}\right)+2 K_{\min } \Delta t\left\|\mathcal{A} \phi^{n+1}\right\|_{\Omega_{p}}^{2} \\
\leq & \frac{K_{\min } \Delta t}{2}\left\|\mathcal{A} \phi^{n+1}\right\|_{\Omega_{p}}^{2}+\frac{4 \Delta t}{K_{\min }}\left\|\mathcal{A} \mathbf{u}^{n}\right\|_{\Omega_{f}}^{2}+\frac{4 \Delta t}{K_{\min }}\left\|g_{p}\left(t_{n+1}\right)\right\|_{\Omega_{p}}^{2} .
\end{aligned}
$$

Summing over the above inequality, we have

$$
\begin{aligned}
& 2 S_{0}\left\|\phi^{n+1}\right\|_{H_{p}}^{2}+2 S_{0} \sum_{i=0}^{n}\left\|\phi^{i+1}-\phi^{i}\right\|_{H_{p}}^{2}+\frac{3}{2} \Delta t \sum_{i=0}^{n}\left\|\mathbf{K}^{1 / 2} \mathcal{A} \phi^{i+1}\right\|_{\Omega_{p}}^{2} \\
\leq & \frac{4 \Delta t}{K_{\min }} \sum_{i=0}^{n}\left\|\mathcal{A} \mathbf{u}^{i}\right\|_{\Omega_{f}}^{2}+\frac{4 \Delta t}{K_{\min }} \sum_{i=0}^{n}\left\|g_{p}\left(t_{i+1}\right)\right\|_{\Omega_{p}}^{2} .
\end{aligned}
$$

Combining (3.15) and (3.17), we arrive at

$$
\begin{aligned}
& \left\|\mathbf{u}^{n+1}\right\|_{H_{f}}^{2}+2 S_{0}\left\|\phi^{n+1}\right\|_{H_{p}}^{2}+\sum_{i=1}^{n}\left(\left\|\mathbf{u}^{i+1}-\mathbf{u}^{i}\right\|_{H_{f}}^{2}+2 S_{0}\left\|\phi^{i+1}-\phi^{i}\right\|_{H_{p}}^{2}\right) \\
& \quad+\frac{3}{2} \Delta t \sum_{i=1}^{n}\left(v\left\|\mathcal{A} \mathbf{u}^{i+1}\right\|_{\Omega_{f}}^{2}+K_{\min }\left\|\mathcal{A} \phi^{i+1}\right\|_{\Omega_{p}}^{2}\right)+2 \theta \Delta t\left\|\mathcal{A} \mathbf{u}^{n+1}\right\|_{\Omega_{f}}^{2} \\
& \quad+2 \theta \Delta t \sum_{i=1}^{n}\left\|\mathcal{A}\left(\mathbf{u}^{i+1}-\mathbf{u}^{i}\right)\right\|_{\Omega_{f}}^{2} \\
& \leq 4 \Delta t v^{-1} \sum_{i=1}^{n}\left\|f\left(t_{i+1}\right)\right\|_{\Omega_{f}}^{2}+4 g \Delta t v^{-1} \sum_{i=1}^{n}\left\|\mathcal{A} \phi^{i}\right\|_{\Omega_{f}}^{2}+4 v^{-1} \Delta t \sum_{i=1}^{n}\left\|\mathbf{u}^{i}\right\|_{H^{2}\left(\Omega_{f}\right)}^{2}\left\|\mathbf{u}^{i}\right\|_{H_{f}}^{2} \\
& \quad+\frac{4 \Delta t}{K_{\min }} \sum_{i=0}^{n}\left\|\mathcal{A} \mathbf{u}^{i}\right\|_{\Omega_{f}}^{2}+\frac{4 \Delta t}{K_{\min }} \sum_{i=0}^{n}\left\|g_{p}\left(t_{i+1}\right)\right\|_{\Omega_{p}}^{2} .
\end{aligned}
$$

Using Gronwall's lemma, it yields

$$
\begin{aligned}
& \left\|\mathbf{u}^{n+1}\right\|_{H_{f}}^{2}+2 S_{0}\left\|\phi^{n+1}\right\|_{H_{p}}^{2}+\sum_{i=1}^{n}\left(\left\|\mathbf{u}^{i+1}-\mathbf{u}^{i}\right\|_{H_{f}}^{2}+2 S_{0}\left\|\phi^{i+1}-\phi^{i}\right\|_{H_{p}}^{2}\right) \\
& \quad+\frac{3}{2} \Delta t \sum_{i=1}^{n}\left(v\left\|\mathcal{A} \mathbf{u}^{i+1}\right\|_{\Omega_{f}}^{2}+K_{\min }\left\|\mathcal{A} \phi^{i+1}\right\|_{\Omega_{p}}^{2}\right)+2 \theta \Delta t\left\|\mathcal{A} \mathbf{u}^{n+1}\right\|_{\Omega_{f}}^{2} \\
& +2 \theta \Delta t \sum_{i=1}^{n}\left\|\mathcal{A}\left(\mathbf{u}^{i+1}-\mathbf{u}^{i}\right)\right\|_{\Omega_{f}}^{2}
\end{aligned}
$$




$$
\begin{aligned}
& \leq \exp \left(4 g \Delta t v^{-1} \sum_{i=1}^{n}\left\|\mathbf{u}^{i}\right\|_{H^{2}\left(\Omega_{f}\right)}^{2}+4 v^{-1} T+\frac{4 T}{K_{\min }}\right) \\
& \quad \times\left(4 \Delta t v^{-1} \sum_{i=1}^{n}\left\|f\left(t_{i+1}\right)\right\|_{\Omega_{f}}^{2}+\frac{4 \Delta t}{K_{\min }} \sum_{i=0}^{n}\left\|g_{p}\left(t_{i+1}\right)\right\|_{\Omega_{p}}^{2}\right) .
\end{aligned}
$$

Step 3: we give the proof of (3.3) under the induction assumption.

Define $d_{t} \mathbf{u}^{n+1}=\frac{\mathbf{u}^{n+1}-\mathbf{u}^{n}}{\Delta t}$. Letting $\mathbf{v}=2 \Delta t d_{t} \mathbf{u}^{n+1}$ in (3.14) and using $\left(\nabla \cdot \mathbf{u}^{n}, q\right)=0, \forall q \in M$, it follows that

$$
\begin{gathered}
2 \Delta t\left\|d_{t} \mathbf{u}^{n+1}\right\|_{\Omega_{f}}^{2}+v\left\|\mathbf{u}^{n+1}\right\|_{H_{f}}^{2}-v\left\|\mathbf{u}^{n}\right\|_{H_{f}}^{2}+\frac{\alpha \sqrt{v g}}{\sqrt{K_{\max }}}\left(\left\|\mathbf{u}^{n+1} \cdot \tau\right\|_{\Gamma}^{2}-\left\|\mathbf{u}^{n} \cdot \tau\right\|_{\Gamma}^{2}\right) \\
+\frac{\alpha \sqrt{v g}}{\sqrt{K_{\max }}}\left\|\mathbf{u}^{n+1} \cdot \tau-\mathbf{u}^{n} \cdot \tau\right\|_{\Gamma}^{2}+v\left\|\mathbf{u}^{n+1}-\mathbf{u}^{n}\right\|_{H_{f}}^{2}+\theta\left\|\mathbf{u}^{n+1}-\mathbf{u}^{n}\right\|_{H_{f}}^{2} \\
+2 \Delta t B\left(\mathbf{u}^{n}, \mathbf{u}^{n}, d_{t} \mathbf{u}^{n+1}\right)+2 \Delta t c_{\Gamma}\left(\phi^{n}, d_{t} \mathbf{u}^{n+1}\right)=2 \Delta t\left(f\left(t_{n+1}\right), d_{t} \mathbf{u}^{n+1}\right) \Omega_{f} .
\end{gathered}
$$

Using (2.9), we have

$$
\begin{aligned}
2 \Delta t B\left(\mathbf{u}^{n}, \mathbf{u}^{n}, d_{t} \mathbf{u}^{n+1}\right) & \leq C \Delta t\left\|\mathbf{u}^{n}\right\|_{H^{2}\left(\Omega_{f}\right)}\left\|\mathbf{u}^{n}\right\|_{H_{f}}\left\|d_{t} \mathbf{u}^{n+1}\right\|_{\Omega_{f}} \\
& \leq 4 \Delta t\left\|\mathbf{u}^{n}\right\|_{H^{2}\left(\Omega_{f}\right)}^{2}\left\|\mathbf{u}^{n}\right\|_{H_{f}}^{2}+\frac{\Delta t}{4}\left\|d_{t} \mathbf{u}^{n+1}\right\|_{\Omega_{f}}^{2} .
\end{aligned}
$$

We also get

$$
\begin{aligned}
& 2 \Delta t c_{\Gamma}\left(\phi^{n}, d_{t} \mathbf{u}^{n+1}\right)=2 c_{\Gamma}\left(\phi^{n}, \mathbf{u}^{n+1}-\mathbf{u}^{n}\right) \\
\leq & C\left\|\nabla \mathbf{K}^{1 / 2} \phi^{n}\right\|_{\Omega_{p}}\left\|\mathbf{u}^{n+1}-\mathbf{u}^{n}\right\|_{H_{f}} \\
\leq & \left\|\nabla \mathbf{K}^{1 / 2} \phi^{n}\right\|_{\Omega_{p}}^{2}+v\left\|\mathbf{u}^{n+1}-\mathbf{u}^{n}\right\|_{H_{f}}^{2} .
\end{aligned}
$$

Using Cauchy-Schwarz inequality, we deduce

$$
\begin{aligned}
& 2 \Delta t\left(f\left(t_{n+1}\right), d_{t} \mathbf{u}^{n+1}\right) \leq 2 \Delta t\left\|f\left(t_{n+1}\right)\right\|_{\Omega_{f}}\left\|d_{t} \mathbf{u}^{n+1}\right\|_{\Omega_{f}} \\
\leq & 4 \Delta t\left\|f\left(t_{n+1}\right)\right\|_{\Omega_{f}}^{2}+\frac{\Delta t}{4}\left\|d_{t} \mathbf{u}^{n+1}\right\|_{\Omega_{f}}^{2} .
\end{aligned}
$$

Then, it follows by

$$
\begin{gathered}
\Delta t\left\|d_{t} \mathbf{u}^{n+1}\right\|_{\Omega_{f}}^{2}+v\left\|\mathbf{u}^{n+1}\right\|_{H_{f}}^{2}-v\left\|\mathbf{u}^{n}\right\|_{H_{f}}^{2}+\frac{\alpha \sqrt{\nu g}}{\sqrt{K_{\max }}}\left(\left\|\mathbf{u}^{n+1} \cdot \tau\right\|_{\Gamma}^{2}-\left\|\mathbf{u}^{n} \cdot \tau\right\|_{\Gamma}^{2}\right) \\
+\frac{\alpha \sqrt{v g}}{\sqrt{K_{\max }}}\left\|\mathbf{u}^{n+1} \cdot \tau-\mathbf{u}^{n} \cdot \tau\right\|_{\Gamma}^{2}+\theta\left\|\mathbf{u}^{n+1}-\mathbf{u}^{n}\right\|_{H_{f}}^{2} \\
\leq 4 \Delta t\left\|\mathcal{A} \mathbf{u}^{n}\right\|_{\Omega_{f}}^{2}\left\|\mathbf{u}^{n}\right\|_{H_{f}}^{2}+\left\|\nabla \phi^{n}\right\|_{\Omega_{p}}^{2}+4 \Delta t\left\|f\left(t_{n+1}\right)\right\|_{\Omega_{f}}^{2} .
\end{gathered}
$$


Summing it over all $n$, there holds

$$
\begin{aligned}
& \Delta t \sum_{i=0}^{n}\left\|d_{t} \mathbf{u}^{i+1}\right\|_{\Omega_{f}}^{2}+v\left\|\mathbf{u}^{n+1}\right\|_{H_{f}}^{2}+\frac{\alpha \sqrt{v g}}{\sqrt{K_{\max }}}\left\|\mathbf{u}^{n+1} \cdot \tau\right\|_{\Gamma}^{2} \\
& \quad+\sum_{i=0}^{n} \frac{\alpha \sqrt{v g}}{\sqrt{K_{\max }}}\left\|\mathbf{u}^{i+1} \cdot \tau-\mathbf{u}^{i} \cdot \tau\right\|_{\Gamma}^{2}+\theta \sum_{i=0}^{n}\left\|\mathbf{u}^{i+1}-\mathbf{u}^{i}\right\|_{H_{f}}^{2} \\
& \leq \exp \left(4 \Delta t \sum_{i=0}^{n}\left\|\mathcal{A} \mathbf{u}^{i}\right\|_{\Omega_{f}}^{2}\right)\left(\sum_{i=0}^{n}\left\|\nabla \phi^{i}\right\|_{\Omega_{p}}^{2}+4 \Delta t \sum_{i=0}^{n}\left\|f\left(t_{i+1}\right)\right\|_{\Omega_{f}}^{2}\right) .
\end{aligned}
$$

Via (3.14), we have

$$
\begin{aligned}
& 2(v+\theta)\left\|\mathcal{A} \mathbf{u}^{n+1}\right\|_{\Omega_{f}} \\
& \leq\left\|d_{t} \mathbf{u}^{n+1}\right\|_{0}+2 \theta\left\|\mathcal{A} \mathbf{u}^{n}\right\|_{\Omega_{f}}^{2}+4\left\|f\left(t_{n+1}\right)\right\|_{\Omega_{f}}^{2} \\
& \quad+4 g\left\|\mathcal{A} \phi^{n}\right\|_{\Omega_{p}}^{2}+4\left\|\mathbf{u}^{n}\right\|_{H^{2}\left(\Omega_{f}\right)}\left\|\mathbf{u}^{n}\right\|_{H_{f}}
\end{aligned}
$$

It means that

$$
\begin{aligned}
& \quad 2(v+\theta) \Delta t \sum_{i=0}^{n}\left\|\mathcal{A} \mathbf{u}^{i+1}\right\|_{\Omega_{f}} \\
& \leq \tau \sum_{i=0^{n}} \tau \sum_{i=0^{n}}\left\|d_{t} \mathbf{u}^{n+1}\right\|_{0}+2 \theta\left\|\mathcal{A} \mathbf{u}^{i}\right\|_{\Omega_{f}}^{2}+4 \tau \sum_{i=0^{n}}\left\|f\left(t_{i+1}\right)\right\|_{\Omega_{f}}^{2} \\
& \quad+4 g \tau \sum_{i=0^{n}}\left\|\mathcal{A} \phi^{i}\right\|_{\Omega_{p}}^{2}+4 \tau \sum_{i=0^{n}}\left\|\mathbf{u}^{i}\right\|_{H^{2}\left(\Omega_{f}\right)}\left\|\mathbf{u}^{i}\right\|_{H_{f}} \leq C .
\end{aligned}
$$

So we finish the proof.

\section{Error analysis}

We define

$$
\begin{aligned}
& \mathbf{e}^{n+1}=\mathbf{u}\left(t_{n+1}\right)-\mathbf{u}^{n+1}, \\
& \mathbf{e}^{n+1 / 2}=\mathbf{u}\left(t_{n+1}\right)-\mathbf{u}^{n+1 / 2}, \\
& \eta^{n+1}=\phi\left(t_{n+1}\right)-\phi^{n+1} .
\end{aligned}
$$

Theorem 4.1 (The non-optimal error estimates). If $u, u_{t} \in H^{2}\left(\Omega_{f}\right), \phi, \phi_{t} \in H^{2}\left(\Omega_{p}\right)$ and $p \in H^{1}\left(\Omega_{f}\right)$, there exists $k_{0}>0$ such that when $\Delta t \leq k_{0}$,

$$
\begin{aligned}
& \left\|\mathbf{e}^{n+1}\right\|_{\Omega_{f}}^{2}+\sum_{i=0}^{n}\left\|\mathbf{e}^{i+1}-\mathbf{e}^{i+1 / 2}\right\|_{\Omega_{f}}^{2}+\sum_{i=0}^{n}\left\|\mathbf{e}^{i+1 / 2}-\mathbf{e}^{i}\right\|_{\Omega_{f}}^{2}+\Delta t v \sum_{i=0}^{n}\left\|\mathbf{e}^{i+1 / 2}\right\|_{H_{f}}^{2} \\
& +\frac{\Delta t \alpha \sqrt{\nu g}}{\sqrt{K_{\max }}} \sum_{i=0}^{n}\left(\left\|\mathbf{e}^{i+1} \cdot \tau\right\|_{\Gamma}^{2}+\left\|\mathbf{e}^{i+1} \cdot \tau-\mathbf{e}^{i+1 / 2} \cdot \tau\right\|_{\Gamma}^{2}\right)
\end{aligned}
$$




$$
\begin{aligned}
& +\theta \Delta t\left(\left\|\mathbf{e}^{n+1}\right\|_{H_{f}}^{2}+\sum_{i=0}^{n}\left\|\mathbf{e}^{i+1}-\mathbf{e}^{i+1 / 2}\right\|_{H_{f}}^{2}\right)+\Delta t v \sum_{i=0}^{n}\left(\left\|\mathbf{e}^{i+1}\right\|_{H_{f}}^{2}+\left\|\mathbf{e}^{i+1}-\mathbf{e}^{i+1 / 2}\right\|_{H_{f}}^{2}\right) \\
& +S_{0}\left(\left\|\eta^{n+1}\right\|_{\Omega_{p}}^{2}+\sum_{i=0}^{n}\left\|\eta^{i+1}-\eta^{i}\right\|_{\Omega_{p}}^{2}\right)+\Delta t \sum_{i=0}^{n}\left\|\mathbf{K}^{1 / 2} \nabla \eta^{i+1}\right\|_{\Omega_{p}}^{2} \leq C \Delta t
\end{aligned}
$$

where $C$ is a positive constant independent of $\Delta t$.

Proof. Subtracting (2.13) from (2.6) with $\psi=0$, we have

$$
\begin{aligned}
& \left(\frac{\mathbf{e}^{n+1 / 2}-\mathbf{e}^{n}}{\Delta t}, \mathbf{v}\right)_{\Omega_{f}}+a_{f}\left(\mathbf{e}^{n+1 / 2}, \mathbf{v}\right)+\theta\left(\nabla \mathbf{e}^{n+1 / 2}, \nabla \mathbf{v}\right)_{\Omega_{f}} \\
& -\theta\left(\nabla\left(\mathbf{u}\left(t_{n+1}\right)-\mathbf{u}^{n}\right), \nabla \mathbf{v}\right)_{\Omega_{f}}+B\left(\mathbf{u}\left(t_{n+1}\right), \mathbf{u}\left(t_{n+1}\right), \mathbf{v}\right)-B\left(\mathbf{u}^{n}, \mathbf{u}^{n}, \mathbf{v}\right) \\
= & b\left(\mathbf{v}, p\left(t_{n+1}\right)\right)+\left(R^{n}, \mathbf{v}\right)_{\Omega_{f}}+c_{\Gamma}\left(\phi\left(t_{n+1}\right)-\phi^{n}, \mathbf{v}\right),
\end{aligned}
$$

where $R^{n}=\mathbf{u}_{t}\left(t_{n+1}\right)-\frac{\mathbf{u}\left(t_{n+1}\right)-\mathbf{u}\left(t_{n}\right)}{\Delta t}$.

Letting $\mathbf{v}=2 \Delta t \mathbf{e}^{n+1 / 2}$, we can deduce

$$
\begin{aligned}
& \left\|\mathbf{e}^{n+1 / 2}\right\|_{\Omega_{f}}^{2}-\left\|\mathbf{e}^{n}\right\|_{\Omega_{f}}^{2}+\left\|\mathbf{e}^{n+1 / 2}-\mathbf{e}^{n}\right\|_{\Omega_{f}}^{2}+2 \Delta t v\left\|\mathbf{e}^{n+1 / 2}\right\|_{H_{f}}^{2}+\frac{2 \Delta t \alpha \sqrt{v g}}{\sqrt{K_{\max }}}\left\|\mathbf{e}^{n+1 / 2} \cdot \tau\right\|_{\Gamma}^{2} \\
& +\theta \Delta t\left(\left\|\mathbf{e}^{n+1 / 2}\right\|_{H_{f}}^{2}-\left\|\mathbf{e}^{n}\right\|_{H_{f}}^{2}+\left\|\mathbf{e}^{n+1 / 2}-\mathbf{e}^{n}\right\|_{H_{f}}^{2}\right) \\
\leq- & 2 \Delta t B\left(\mathbf{u}\left(t_{n+1}\right), \mathbf{u}\left(t_{n+1}\right), \mathbf{e}^{n+1 / 2}\right)+2 \Delta t B\left(\mathbf{u}^{n}, \mathbf{u}^{n}, \mathbf{e}^{n+1 / 2}\right)+2 \Delta t b\left(\mathbf{e}^{n+1 / 2}, p\left(t_{n+1}\right)\right) \\
& +2 \theta \Delta t\left(\nabla\left(\mathbf{u}\left(t_{n+1}\right)-\mathbf{u}\left(t_{n}\right)\right), \nabla \mathbf{e}^{n+1 / 2}\right)_{\Omega_{f}}+2 \Delta t\left(R^{n}, \mathbf{e}^{n+1 / 2}\right)_{\Omega_{f}} \\
& +2 \Delta t c_{\Gamma}\left(\phi\left(t_{n+1}\right)-\phi^{n}, \mathbf{e}^{n+1 / 2}\right) .
\end{aligned}
$$

Form (2.14), we have

$$
\begin{aligned}
& \left(\frac{\mathbf{e}^{n+1}-\mathbf{e}^{n+1 / 2}}{\Delta t}, \mathbf{v}\right)_{\Omega_{f}}+a_{f}\left(\mathbf{e}^{n+1}-\mathbf{e}^{n+1 / 2}, \mathbf{v}\right) \\
& \quad+\theta\left(\nabla \mathbf{e}^{n+1}-\nabla \mathbf{e}^{n+1 / 2}, \nabla \mathbf{v}\right)_{\Omega_{f}}+b\left(\mathbf{v}, p^{n+1}\right)=0
\end{aligned}
$$

Taking $\mathbf{v}=2 \Delta t \mathbf{e}^{n+1}$ and noticing the fact that $\nabla \cdot \mathbf{e}^{n+1}=0$, we arrive at

$$
\begin{aligned}
& \left\|\mathbf{e}^{n+1}\right\|_{\Omega_{f}}^{2}-\left\|\mathbf{e}^{n+1 / 2}\right\|_{\Omega_{f}}^{2}+\left\|\mathbf{e}^{n+1}-\mathbf{e}^{n+1 / 2}\right\|_{\Omega_{f}}^{2} \\
& +\frac{\Delta t \alpha \sqrt{v g}}{\sqrt{K_{\max }}}\left(\left\|\mathbf{e}^{n+1} \cdot \tau\right\|_{\Gamma}^{2}-\left\|\mathbf{e}^{n+1 / 2} \cdot \tau\right\|_{\Gamma}^{2}+\left\|\mathbf{e}^{n+1} \cdot \tau-\mathbf{e}^{n+1 / 2} \cdot \tau\right\|_{\Gamma}^{2}\right) \\
& +(\theta+v) \Delta t\left(\left\|\mathbf{e}^{n+1}\right\|_{H_{f}}^{2}-\left\|\mathbf{e}^{n+1 / 2}\right\|_{H_{f}}^{2}+\left\|\mathbf{e}^{n+1}-\mathbf{e}^{n+1 / 2}\right\|_{H_{f}}^{2}\right) \leq 0 .
\end{aligned}
$$


Summing the inequalities (4.2) and (4.4), we get

$$
\begin{aligned}
&\left\|\mathbf{e}^{n+1}\right\|_{\Omega_{f}}^{2}-\left\|\mathbf{e}^{n}\right\|_{\Omega_{f}}^{2}+\left\|\mathbf{e}^{n+1}-\mathbf{e}^{n+1 / 2}\right\|_{\Omega_{f}}^{2}+\left\|\mathbf{e}^{n+1 / 2}-\mathbf{e}^{n}\right\|_{\Omega_{f}}^{2}+\Delta t v\left\|\mathbf{e}^{n+1 / 2}\right\|_{H_{f}}^{2} \\
&+\frac{2 \Delta t \alpha}{\sqrt{K_{\max }}}\left\|\mathbf{e}^{n+1 / 2} \cdot \tau\right\|_{\Gamma}^{2}+\frac{\Delta t \alpha \sqrt{v g}}{\sqrt{K_{\max }}}\left(\left\|\mathbf{e}^{n+1} \cdot \tau\right\|_{\Gamma}^{2}-\left\|\mathbf{e}^{n+1 / 2} \cdot \tau\right\|_{\Gamma}^{2}+\left\|\mathbf{e}^{n+1} \cdot \tau-\mathbf{e}^{n+1 / 2} \cdot \tau\right\|_{\Gamma}^{2}\right) \\
&+ \\
&+\theta \Delta t\left(\left\|\mathbf{e}^{n+1}\right\|_{H_{f}}^{2}-\left\|\mathbf{e}^{n}\right\|_{H_{f}}^{2}+\left\|\mathbf{e}^{n+1}-\mathbf{e}^{n+1 / 2}\right\|_{H_{f}}^{2}\right)+\Delta t v\left(\left\|\mathbf{e}^{n+1}\right\|_{H_{f}}^{2}+\left\|\mathbf{e}^{n+1}-\mathbf{e}^{n+1 / 2}\right\|_{H_{f}}^{2}\right) \\
& \leq- 2 \Delta t B\left(\mathbf{u}\left(t_{n+1}\right), \mathbf{u}\left(t_{n+1}\right), \mathbf{e}^{n+1 / 2}\right)+2 \Delta t B\left(\mathbf{u}^{n}, \mathbf{u}^{n}, \mathbf{e}^{n+1 / 2}\right)+2 \Delta t b\left(\mathbf{e}^{n+1 / 2}, p\left(t_{n+1}\right)\right) \\
&+2 \theta \Delta t\left(\nabla\left(\mathbf{u}\left(t_{n+1}\right)-\mathbf{u}\left(t_{n}\right)\right), \nabla \mathbf{e}^{n+1 / 2}\right)_{\Omega_{f}}+2 \Delta t\left(R^{n}, \mathbf{e}^{n+1 / 2}\right)_{\Omega_{f}} \\
&+2 \Delta t c_{\Gamma}\left(\phi\left(t_{n+1}\right)-\phi^{n}, \mathbf{e}^{n+1 / 2}\right) .
\end{aligned}
$$

Using (2.9), we deduce

$$
\begin{aligned}
&-2 \Delta t B\left(\mathbf{u}\left(t_{n+1}\right), \mathbf{u}\left(t_{n+1}\right), \mathbf{e}^{n+1 / 2}\right)+2 \Delta t B\left(\mathbf{u}^{n}, \mathbf{u}^{n}, \mathbf{e}^{n+1 / 2}\right) \\
&=2 \Delta {\left[-B\left(\mathbf{e}^{n}, \mathbf{u}^{n}, \mathbf{e}^{n+1 / 2}\right)-B\left(\mathbf{u}\left(t_{n+1}\right)-\mathbf{u}\left(t_{n}\right), \mathbf{u}\left(t_{n}\right), \mathbf{e}^{n+1 / 2}\right)-B\left(\mathbf{u}\left(t_{n}\right), \mathbf{e}^{n}, \mathbf{e}^{n+1 / 2}\right)\right.} \\
&\left.-B\left(\mathbf{u}\left(t_{n+1}\right), \mathbf{u}\left(t_{n+1}\right)-\mathbf{u}\left(t_{n}\right), \mathbf{e}^{n+1 / 2}\right)\right] \\
& \leq 2 c_{1} \Delta t\left\|\mathbf{e}^{n}\right\|_{\Omega_{f}}\left\|\mathbf{u}^{n}\right\|_{H^{2}\left(\Omega_{f}\right)}\left\|\nabla \mathbf{e}^{n+1 / 2}\right\|_{\Omega_{f}}+2 \Delta t c_{1}\left\|\mathbf{u}\left(t_{n+1}\right)-\mathbf{u}\left(t_{n}\right)\right\|_{\Omega_{f}}\left\|\mathbf{u}\left(t_{n}\right)\right\|_{H^{2}\left(\Omega_{f}\right)}\left\|\nabla \mathbf{e}^{n+1 / 2}\right\|_{\Omega_{f}} \\
&+2 \Delta t c_{1}\left\|\mathbf{u}\left(t_{n}\right)\right\|_{H^{2}\left(\Omega_{f}\right.}\left\|\mathbf{e}^{n}\right\|_{\Omega_{f}}\left\|\nabla \mathbf{e}^{n+1 / 2}\right\|_{\Omega_{f}} \\
&+2 \Delta t\left\|\mathcal{A} \mathbf{u}\left(t_{n+1}\right)\right\|_{\Omega_{f}}\left\|\mathbf{u}\left(t_{n+1}\right)-\mathbf{u}\left(t_{n}\right)\right\|_{\Omega_{f}}\left\|\mathbf{e}^{n+1 / 2}\right\|_{H_{f}} \\
& \leq 8 v^{-1} \Delta t c_{1}^{2}\left\|\mathbf{u}^{n}\right\|_{H^{2}\left(\Omega_{f}\right)}^{2}\left\|\mathbf{e}^{n}\right\|_{\Omega_{f}}^{2}+8 \Delta t c_{1}^{2}\left\|\mathbf{u}\left(t_{n}\right)\right\|_{H^{2}\left(\Omega_{f}\right)}^{2}\left\|\mathbf{u}\left(t_{n+1}\right)-\mathbf{u}\left(t_{n}\right)\right\|_{\Omega_{f}}^{2} \\
& \quad+8 \Delta t c_{1}^{2}\left\|\mathbf{u}\left(t_{n}\right)\right\|_{H^{2}\left(\Omega_{f}\right.}^{2}\left\|\mathbf{e}^{n}\right\|_{\Omega_{f}}^{2}+8 c_{1}^{2} \Delta t\left\|\mathcal{A} \mathbf{u}\left(t_{n+1}\right)\right\|_{\Omega_{f}}^{2}\left\|\mathbf{u}\left(t_{n+1}\right)-\mathbf{u}\left(t_{n}\right)\right\|_{\Omega_{f}}^{2} \\
& \quad+\frac{v \Delta t}{4}\left\|\mathbf{e}^{n+1 / 2}\right\|_{H_{f}}^{2} \\
& \leq 8 v^{-1} \Delta t c_{1}^{2}\left(\left\|\mathbf{u}^{n}\right\|_{H^{2}\left(\Omega_{f}\right)}^{2}+\left\|\mathbf{u}\left(t_{n}\right)\right\|_{H^{2}\left(\Omega_{f}\right)}^{2}\right)\left\|\mathbf{e}^{n}\right\|_{\Omega_{f}}^{2} \\
& \quad+C \Delta t^{3}\left\|\mathbf{u}\left(t_{n}\right)\right\|_{H^{2}\left(\Omega_{f}\right)}^{2}\left\|\mathbf{u}_{t}\left(t_{n+1}\right)\right\|_{\Omega_{f}}^{2}+\frac{v \Delta t}{8}\left\|\mathbf{e}^{n+1 / 2}\right\|_{H_{f}}^{2} .
\end{aligned}
$$

Using Cauchy-Schwarz inequality and Taylor's formula, we deduce

$$
\begin{aligned}
& 2 \theta \Delta t\left(\nabla\left(\mathbf{u}\left(t_{n+1}\right)-\mathbf{u}\left(t_{n}\right)\right), \nabla \mathbf{e}^{n+1 / 2}\right)_{\Omega_{f}} \\
\leq & 2 \theta \Delta t\left\|\mathbf{u}\left(t_{n+1}\right)-\mathbf{u}\left(t_{n}\right)\right\|_{H_{f}}\left\|\mathbf{e}^{n+1 / 2}\right\|_{H_{f}} \\
\leq & C \theta^{2} \Delta t^{3}\left\|\mathbf{u}_{t}\left(t_{n+1}\right)\right\|_{H_{f}}^{2}+\frac{v \Delta t}{8}\left\|\mathbf{e}^{n+1 / 2}\right\|_{H_{f}}^{2} .
\end{aligned}
$$

Using Taylor's Formula, we have

$$
2 \Delta t\left(R^{n}, \mathbf{e}^{n+1 / 2}\right)_{\Omega_{f}} \leq C \Delta t^{3}+\frac{v \Delta t}{8}\left\|\mathbf{e}^{n+1 / 2}\right\|_{H_{f}}^{2} .
$$


Via (3.1), we can get

$$
\begin{aligned}
& 2 \Delta t c_{\Gamma}\left(\phi\left(t_{n+1}\right)-\phi^{n}, \mathbf{e}^{n+1 / 2}\right) \leq 2 C_{2} g \Delta t\left\|\phi\left(t_{n+1}\right)-\phi^{n}\right\|_{H_{p}}\left\|\mathbf{e}^{n+1 / 2}\right\|_{H_{f}} \\
\leq & 2 C_{2} g \Delta t\left(\left\|\phi\left(t_{n+1}\right)-\phi\left(t_{n}\right)\right\|_{H_{p}}+\left\|\phi\left(t_{n}\right)-\phi^{n}\right\|_{H_{p}}\right)\left\|\mathbf{e}^{n+1 / 2}\right\|_{H_{f}} \\
\leq & C g \Delta t^{3}+\frac{C \Delta t}{K_{\min }}\left\|\mathbf{K}^{1 / 2} \nabla \eta^{n}\right\|_{\Omega_{p}}^{2}+\frac{v \Delta t}{8}\left\|\mathbf{e}^{n+1 / 2}\right\|_{H_{f}}^{2} .
\end{aligned}
$$

Noting $\nabla \cdot \mathbf{e}^{n}=0$ and using Cauchy-Schwarz inequality, we have

$$
\begin{aligned}
& 2 \Delta t b\left(\mathbf{e}^{n+1 / 2}, p\left(t_{n}\right)\right)=2 \Delta t b\left(\mathbf{e}^{n+1 / 2}-\mathbf{e}^{n}, p\left(t_{n}\right)\right) \\
\leq & 2 \Delta t\left\|\mathbf{e}^{n+1 / 2}-\mathbf{e}^{n}\right\|_{\Omega_{f}}\left\|p\left(t_{n}\right)\right\|_{H_{f}} \leq \frac{1}{2}\left\|\mathbf{e}^{n+1 / 2}-\mathbf{e}^{n}\right\|_{\Omega_{f}}+2 \Delta t^{2}\left\|p\left(t_{n}\right)\right\|_{H_{f}}^{2} .
\end{aligned}
$$

Combining these inequalities with (4.5), we arrive at

$$
\begin{aligned}
& \left\|\mathbf{e}^{n+1}\right\|_{\Omega_{f}}^{2}-\left\|\mathbf{e}^{n}\right\|_{\Omega_{f}}^{2}+\left\|\mathbf{e}^{n+1}-\mathbf{e}^{n+1 / 2}\right\|_{\Omega_{f}}^{2}+\Delta t v\left\|\mathbf{e}^{n+1 / 2}\right\|_{H_{f}}^{2}+\frac{2 \Delta t \alpha}{\sqrt{K_{\max }}}\left\|\mathbf{e}^{n+1 / 2} \cdot \tau\right\|_{\Gamma}^{2} \\
& +\frac{\alpha \sqrt{\nu g}}{\sqrt{K_{\max }}}\left(\left\|\mathbf{e}^{n+1} \cdot \tau\right\|_{\Gamma}^{2}-\left\|\mathbf{e}^{n+1 / 2} \cdot \tau\right\|_{\Gamma}^{2}+\left\|\mathbf{e}^{n+1} \cdot \tau-\mathbf{e}^{n+1 / 2} \cdot \tau\right\|_{\Gamma}^{2}\right) \\
& \quad+\theta \Delta t\left(\left\|\mathbf{e}^{n+1}\right\|_{H_{f}}^{2}-\left\|\mathbf{e}^{n}\right\|_{H_{f}}^{2}+\left\|\mathbf{e}^{n+1}-\mathbf{e}^{n+1 / 2}\right\|_{H_{f}}^{2}\right)+\Delta t v\left(\left\|\mathbf{e}^{n+1}\right\|_{H_{f}}^{2}+\left\|\mathbf{e}^{n+1}-\mathbf{e}^{n+1 / 2}\right\|_{H_{f}}^{2}\right) \\
& \leq 8 v^{-1} \Delta t c_{1}^{2}\left(\left\|\mathbf{u}^{n}\right\|_{H^{2}\left(\Omega_{f}\right)}^{2}+\left\|\mathbf{u}\left(t_{n}\right)\right\|_{H^{2}\left(\Omega_{f}\right)}^{2}\right)\left\|\mathbf{e}^{n}\right\|_{\Omega_{f}}^{2}+C \Delta t^{3}\left\|\mathbf{u}\left(t_{n}\right)\right\|_{H^{2}\left(\Omega_{f}\right)}^{2}\left\|\mathbf{u}_{t}\left(t_{n+1}\right)\right\|_{\Omega_{f}}^{2} \\
& \quad+C \theta^{2} \Delta t^{3}+2 \Delta t^{2}\left\|p\left(t_{n}\right)\right\|_{H_{f}}^{2}+\frac{C \Delta t}{K_{\min }}\left\|\mathbf{K}^{1 / 2} \nabla \eta^{n}\right\|_{\Omega_{p}}^{2}+\frac{v \Delta t}{2}\left\|\mathbf{e}^{n+1 / 2}\right\|_{H_{f}}^{2} .
\end{aligned}
$$

Subtracting (2.15) for the weak form of (2.3), we have

$$
g S_{0}\left(\frac{\eta^{n+1}-\eta^{n}}{\Delta t}, \psi\right)_{\Omega_{p}}+2 g \Delta t\left(\mathbf{K} \nabla \eta^{n+1}, \nabla \psi\right)_{\Omega_{p}}+2 \Delta t c_{\Gamma}\left(\psi, \mathbf{u}\left(t_{n+1}\right)-\mathbf{u}^{n}\right)=0 .
$$

It follows from the above equality, with $\psi=2 \Delta t \eta^{n+1}$, that

$$
\begin{aligned}
& g S_{0}\left(\left\|\eta^{n+1}\right\|_{\Omega_{p}}^{2}-\left\|\eta^{n}\right\|_{\Omega_{p}}+\left\|\eta^{n+1}-\eta^{n}\right\|_{\Omega_{p}}^{2}\right)+2 g \Delta t\left\|\mathbf{K}^{1 / 2} \nabla \eta^{n+1}\right\|_{\Omega_{p}} \\
= & -2 \Delta t c_{\Gamma}\left(\eta^{n+1}, \mathbf{u}\left(t_{n+1}\right)-\mathbf{u}^{n}\right) .
\end{aligned}
$$

Using (3.1), we can deduce

$$
2 \Delta t c_{\Gamma}\left(\eta^{n+1}, \mathbf{u}\left(t_{n+1}\right)-\mathbf{u}^{n}\right) \leq C g \Delta t^{3}+C g \Delta t\left\|\mathbf{e}^{n}\right\|_{H_{f}}^{2}+\frac{g \Delta t}{4}\left\|\mathbf{K}^{1 / 2} \nabla \eta^{n+1}\right\|_{\Omega_{p}}^{2} .
$$

Then, we have

$$
\begin{aligned}
& g S_{0}\left(\left\|\eta^{n+1}\right\|_{\Omega_{p}}^{2}-\left\|\eta^{n}\right\|_{\Omega_{p}}^{2}+\left\|\eta^{n+1}-\eta^{n}\right\|_{\Omega_{p}}^{2}\right)+2 g \Delta t\left\|\mathbf{K}^{1 / 2} \nabla \eta^{n+1}\right\|_{\Omega_{p}} \\
\leq & C g \Delta t^{3}+C g \Delta t\left\|\mathbf{e}^{n}\right\|_{H_{f}}^{2}+\frac{g \Delta t}{4}\left\|\mathbf{K}^{1 / 2} \nabla \eta^{n+1}\right\|_{\Omega_{p}}^{2} .
\end{aligned}
$$


Summing (4.11) with (4.13), we get

$$
\begin{aligned}
& \left\|\mathbf{e}^{n+1}\right\|_{\Omega_{f}}^{2}-\left\|\mathbf{e}^{n}\right\|_{\Omega_{f}}^{2}+\left\|\mathbf{e}^{n+1}-\mathbf{e}^{n+1 / 2}\right\|_{\Omega_{f}}^{2}+\Delta t v\left\|\mathbf{e}^{n+1 / 2}\right\|_{H_{f}}^{2} \\
& \quad+\frac{\Delta t \alpha \sqrt{v g}}{\sqrt{K_{\max }}}\left(\left\|\mathbf{e}^{n+1} \cdot \tau\right\|_{\Gamma}^{2}+\left\|\mathbf{e}^{n+1} \cdot \tau-\mathbf{e}^{n+1 / 2} \cdot \tau\right\|_{\Gamma}^{2}\right) \\
& \quad+\theta \Delta t\left(\left\|\mathbf{e}^{n+1}\right\|_{H_{f}}^{2}-\left\|\mathbf{e}^{n}\right\|_{H_{f}}^{2}+\left\|\mathbf{e}^{n+1}-\mathbf{e}^{n+1 / 2}\right\|_{H_{f}}^{2}\right) \\
& \quad+\Delta t v\left(\left\|\mathbf{e}^{n+1}\right\|_{H_{f}}^{2}+\left\|\mathbf{e}^{n+1}-\mathbf{e}^{n+1 / 2}\right\|_{H_{f}}^{2}\right) \\
& \quad+g S_{0}\left(\left\|\eta^{n+1}\right\|_{\Omega_{p}}^{2}-\left\|\eta^{n}\right\|_{\Omega_{p}}^{2}+\left\|\eta^{n+1}-\eta^{n}\right\|_{\Omega_{p}}^{2}\right)+g \Delta t\left\|\mathbf{K}^{1 / 2} \nabla \eta^{n+1}\right\|_{\Omega_{p}} \\
& \leq 8 v^{-1} \Delta t c_{1}^{2}\left(\left\|\mathbf{u}^{n}\right\|_{H^{2}\left(\Omega_{f}\right)}^{2}+\left\|\mathbf{u}\left(t_{n}\right)\right\|_{H^{2}\left(\Omega_{f}\right)}^{2}\right)\left\|^{n}\right\|_{\Omega_{f}}^{2}+C \Delta t^{3}\left\|\mathbf{u}\left(t_{n}\right)\right\|_{H^{2}\left(\Omega_{f}\right)}^{2}\left\|\mathbf{u}_{t}\left(t_{n+1}\right)\right\|_{\Omega_{f}}^{2} \\
& \quad+C \theta^{2} \Delta t^{3}\left\|\mathbf{u}_{t}\left(t_{n+1}\right)\right\|_{H_{f}}^{2}+\frac{v \Delta t}{2}\left\|\mathbf{e}^{n+1 / 2}\right\|_{H_{f}}^{2}+C \Delta t^{2}+C \Delta t\left\|\mathbf{e}^{n}\right\|_{H_{f}}^{2} \\
& \quad+C g \Delta t\left\|\mathbf{K}^{1 / 2} \nabla \eta^{n}\right\|_{\Omega_{p}}^{2} .
\end{aligned}
$$

Summing (4.14) form 0 to $n$ and using Gronwall's lemma twice, we can deduce

$$
\begin{aligned}
& \left\|\mathbf{e}^{n+1}\right\|_{\Omega_{f}}^{2}+\sum_{i=0}^{n}\left\|\mathbf{e}^{i+1}-\mathbf{e}^{i+1 / 2}\right\|_{\Omega_{f}}^{2}+\sum_{i=0}^{n}\left\|\mathbf{e}^{i+1 / 2}-\mathbf{e}^{i}\right\|_{\Omega_{f}}^{2}+\Delta t v \sum_{i=0}^{n}\left\|\mathbf{e}^{i+1 / 2}\right\|_{H_{f}}^{2} \\
& \quad+\frac{\alpha \sqrt{v g}}{\sqrt{K_{\max }}} \sum_{i=0}^{n}\left(\left\|\mathbf{e}^{i+1} \cdot \tau\right\|_{\Gamma}^{2}+\left\|\mathbf{e}^{i+1} \cdot \tau-\mathbf{e}^{i+1 / 2} \cdot \tau\right\|_{\Gamma}^{2}\right) \\
& \quad+\theta \Delta t\left(\left\|\mathbf{e}^{n+1}\right\|_{H_{f}}^{2}+\sum_{i=0}^{n}\left\|\mathbf{e}^{i+1}-\mathbf{e}^{i+1 / 2}\right\|_{H_{f}}^{2}\right)+\Delta t v \sum_{i=0}^{n}\left(\left\|\mathbf{e}^{i+1}\right\|_{H_{f}}^{2}+\left\|\mathbf{e}^{i+1}-\mathbf{e}^{i+1 / 2}\right\|_{H_{f}}^{2}\right) \\
& \quad+S_{0}\left(\left\|\eta^{n+1}\right\|_{\Omega_{p}}^{2}+\sum_{i=0}^{n}\left\|\eta^{i+1}-\eta^{i}\right\|_{\Omega_{p}}^{2}\right)+\Delta t \sum_{i=0}^{n}\left\|\mathbf{K}^{1 / 2} \nabla \eta^{i+1}\right\|_{\Omega_{p}} \leq C \Delta t .
\end{aligned}
$$

So we finish the proof.

Theorem 4.2 (The optimal error estimates). If $u, u_{t} \in H^{2}\left(\Omega_{f}\right), \phi, \phi_{t} \in H^{2}\left(\Omega_{p}\right)$ and $p \in$ $H^{1}\left(\Omega_{f}\right)$, there exist $k_{0}>0$ such that when $\Delta t \leq k_{0}$,

$$
\begin{aligned}
& \left\|\mathbf{e}^{n+1}\right\|_{\Omega_{f}}^{2}+\sum_{i=0}^{n}\left\|\mathbf{e}^{i+1}-\mathbf{e}^{i+1 / 2}\right\|_{\Omega_{f}}^{2}+\sum_{i=0}^{n}\left\|\mathbf{e}^{i+1 / 2}-\mathbf{e}^{i}\right\|_{\Omega_{f}}^{2}+\Delta t(v+\theta) \sum_{i=0}^{n}\left\|\mathbf{e}^{i+1}\right\|_{H_{f}}^{2} \\
& \quad+\frac{\alpha \sqrt{v g}}{\sqrt{K_{\max }}} \sum_{i=0}^{n}\left\|\mathbf{e}^{i+1} \cdot \tau\right\|_{\Gamma}^{2}+\theta \Delta t\left(\left\|\mathbf{e}^{n+1}\right\|_{H_{f}}^{2}+\sum_{i=0}^{n}\left\|\mathbf{e}^{i+1}-\mathbf{e}^{i+1 / 2}\right\|_{H_{f}}^{2}\right) \\
& \quad+\Delta t v \sum_{i=0}^{n}\left(\left\|\mathbf{e}^{i+1}\right\|_{H_{f}}^{2}+\left\|\mathbf{e}^{i+1}-\mathbf{e}^{i+1 / 2}\right\|_{H_{f}}^{2}\right)+g S_{0}\left(\left\|\eta^{n+1}\right\|_{\Omega_{p}}^{2}+\sum_{i=0}^{n}\left\|\eta^{i+1}-\eta^{i}\right\|_{\Omega_{p}}^{2}\right) \\
& \quad+\Delta t \sum_{i=0}^{n}\left\|\mathbf{K}^{1 / 2} \nabla \eta^{i+1}\right\|_{\Omega_{p}} \leq C \Delta t^{2} .
\end{aligned}
$$


Proof. Summing (4.1) and (4.3), we arrive at

$$
\begin{aligned}
& \left(\frac{\mathbf{e}^{n+1}-\mathbf{e}^{n}}{\Delta t}, \mathbf{v}\right)_{\Omega_{f}}+a_{f}\left(\mathbf{e}^{n+1}, \mathbf{v}\right)_{\Omega_{f}}+\theta\left(\nabla \mathbf{e}^{n+1}, \nabla \mathbf{v}\right)_{\Omega_{f}} \\
& -\theta\left(\nabla\left(\mathbf{u}\left(t_{n+1}\right)-\mathbf{u}^{n}\right), \nabla \mathbf{v}\right)_{\Omega_{f}}+B\left(\mathbf{u}\left(t_{n+1}\right), \mathbf{u}\left(t_{n+1}\right), \mathbf{v}\right)-B\left(\mathbf{u}^{n}, \mathbf{u}^{n}, \mathbf{v}\right) \\
= & b\left(\mathbf{v}, p\left(t_{n+1}\right)-p^{n+1}\right)+\left(R^{n}, \mathbf{v}\right)_{\Omega_{f}}+c_{\Gamma}\left(\phi\left(t_{n+1}\right)-\phi^{n}, \mathbf{v}\right) .
\end{aligned}
$$

Letting $\mathbf{v}=2 \Delta t \mathbf{e}^{n+1}$ in (4.15) and noting that $\nabla \cdot \mathbf{e}^{n+1}=0$, we can deduce

$$
\begin{gathered}
\left\|\mathbf{e}^{n+1}\right\|_{\Omega_{f}}^{2}-\left\|\mathbf{e}^{n}\right\|_{\Omega_{f}}^{2}+\left\|\mathbf{e}^{n+1}-\mathbf{e}^{n}\right\|_{\Omega_{f}}^{2}+2 \Delta t(v+\theta)\left\|\mathbf{e}^{n+1}\right\|_{H_{f}}^{2} \\
+\frac{\alpha \sqrt{\nu g}}{\sqrt{K_{\max }}} \sum_{i=0}^{n}\left\|\mathbf{e}^{i+1} \cdot \tau\right\|_{\Gamma}^{2}-2 \Delta t \theta\left(\nabla\left(\mathbf{u}\left(t_{n+1}\right)-\mathbf{u}^{n}\right), \nabla \mathbf{e}^{n+1}\right)_{\Omega_{f}} \\
\quad+2 \Delta t B\left(\mathbf{u}\left(t_{n+1}\right), \mathbf{u}\left(t_{n+1}\right), \mathbf{e}^{n+1}\right)-2 \Delta t B\left(\mathbf{u}^{n}, \mathbf{u}^{n}, \mathbf{e}^{n+1}\right) \\
\leq 2 \Delta t\left(R^{n}, \mathbf{e}^{n+1}\right)_{\Omega_{f}}+2 \Delta t c_{\Gamma}\left(\phi\left(t_{n+1}\right)-\phi^{n}, \mathbf{e}^{n+1}\right) .
\end{gathered}
$$

Using (4.6)-(4.9), we can deduce

$$
\begin{aligned}
& \left\|\mathbf{e}^{n+1}\right\|_{\Omega_{f}}^{2}-\left\|\mathbf{e}^{n}\right\|_{\Omega_{f}}^{2}+\left\|\mathbf{e}^{n+1}-\mathbf{e}^{n}\right\|_{\Omega_{f}}^{2}+2 \Delta t(v+\theta)\left\|\mathbf{e}^{n+1}\right\|_{H_{f}}^{2}+\frac{\alpha \sqrt{v g}}{\sqrt{K_{\max }}} \sum_{i=0}^{n}\left\|\mathbf{e}^{i+1} \cdot \tau\right\|_{\Gamma}^{2} \\
& \leq 8 v^{-1} \Delta t c_{1}^{2}\left(\left\|\mathbf{u}^{n}\right\|_{H^{2}\left(\Omega_{f}\right)}^{2}+\left\|\mathbf{u}\left(t_{n}\right)\right\|_{H^{2}\left(\Omega_{f}\right)}^{2}\right)\left\|\mathbf{e}^{n}\right\|_{\Omega_{f}}^{2}+C \Delta t^{3}\left\|\mathbf{u}\left(t_{n}\right)\right\|_{H^{2}\left(\Omega_{f}\right)}^{2}\left\|\mathbf{u}_{t}\left(t_{n+1}\right)\right\|_{\Omega_{f}}^{2} \\
& \quad+C \theta^{2} \Delta t^{3}+\frac{C \Delta t}{K_{\min }}\left\|\mathbf{K}^{1 / 2} \nabla \eta^{n}\right\|_{\Omega_{p}}^{2}+\frac{v \Delta t}{2}\left\|\mathbf{e}^{n+1 / 2}\right\|_{H_{f}}^{2} .
\end{aligned}
$$

Summing it with (4.13), we can deduce

$$
\begin{aligned}
& \left\|\mathbf{e}^{n+1}\right\|_{\Omega_{f}}^{2}-\left\|\mathbf{e}^{n}\right\|_{\Omega_{f}}^{2}+\left\|\mathbf{e}^{n+1}-\mathbf{e}^{n}\right\|_{\Omega_{f}}^{2}+2 \Delta t(v+\theta)\left\|\mathbf{e}^{n+1}\right\|_{H_{f}}^{2}+\frac{\alpha \sqrt{v g}}{\sqrt{K_{\max }}} \sum_{i=0}^{n}\left\|\mathbf{e}^{i+1} \cdot \tau\right\|_{\Gamma}^{2} \\
& \quad+g S_{0}\left(\left\|\eta^{n+1}\right\|_{\Omega_{p}}^{2}-\left\|\eta^{n}\right\|_{\Omega_{p}}+\left\|\eta^{n+1}-\eta^{n}\right\|_{\Omega_{p}}^{2}\right)+2 g \Delta t\left\|\mathbf{K}^{1 / 2} \nabla \eta^{n+1}\right\|_{\Omega_{p}} \\
& \leq 8 v^{-1} \Delta t c_{1}^{2}\left(\left\|\mathbf{u}^{n}\right\|_{H^{2}\left(\Omega_{f}\right)}^{2}+\left\|\mathbf{u}\left(t_{n}\right)\right\|_{H^{2}\left(\Omega_{f}\right)}^{2}\right)\left\|\mathbf{e}^{n}\right\|_{\Omega_{f}}^{2}+C \Delta t^{3}\left\|\mathbf{u}\left(t_{n}\right)\right\|_{H^{2}\left(\Omega_{f}\right)}^{2}\left\|\mathbf{u}_{t}\left(t_{n+1}\right)\right\|_{\Omega_{f}}^{2} \\
& \quad+C \theta^{2} \Delta t^{3}+\frac{C \Delta t}{K_{\min }}\left\|\mathbf{K}^{1 / 2} \nabla \eta^{n}\right\|_{\Omega_{p}}^{2}+\frac{v \Delta t}{2}\left\|\mathbf{e}^{n+1 / 2}\right\|_{H_{f}}^{2}+C g \Delta t\left\|\mathbf{e}^{n}\right\|_{H_{f}}^{2} \\
& \quad+\frac{g \Delta t}{4}\left\|\mathbf{K}^{1 / 2} \nabla \eta^{n+1}\right\|_{H_{p}}^{2} .
\end{aligned}
$$

Summing it form 0 to $n$ and using Gronwall's lemma twice, we can deduce

$$
\begin{gathered}
\left\|\mathbf{e}^{n+1}\right\|_{\Omega_{f}}^{2}+\sum_{i=0}^{n}\left\|\mathbf{e}^{i+1}-\mathbf{e}^{i+1 / 2}\right\|_{\Omega_{f}}^{2}+\sum_{i=0}^{n}\left\|\mathbf{e}^{i+1 / 2}-\mathbf{e}^{i}\right\|_{\Omega_{f}}^{2}+\Delta t(v+\theta) \sum_{i=0}^{n}\left\|\mathbf{e}^{i+1}\right\|_{H_{f}}^{2} \\
+\frac{\alpha \sqrt{v g}}{\sqrt{K_{\max }}} \sum_{i=0}^{n}\left\|\mathbf{e}^{i+1} \cdot \tau\right\|_{\Gamma}^{2}+\theta \Delta t\left(\left\|\mathbf{e}^{n+1}\right\|_{H_{f}}^{2}+\sum_{i=0}^{n}\left\|\mathbf{e}^{i+1}-\mathbf{e}^{i+1 / 2}\right\|_{H_{f}}^{2}\right)
\end{gathered}
$$




$$
\begin{aligned}
& +\Delta t v \sum_{i=0}^{n}\left(\left\|\mathbf{e}^{i+1}\right\|_{H_{f}}^{2}+\left\|\mathbf{e}^{i+1}-\mathbf{e}^{i+1 / 2}\right\|_{H_{f}}^{2}\right)+g S_{0}\left(\left\|\eta^{n+1}\right\|_{\Omega_{p}}^{2}+\sum_{i=0}^{n}\left\|\eta^{i+1}-\eta^{i}\right\|_{\Omega_{p}}^{2}\right) \\
& +\Delta t \sum_{i=0}^{n}\left\|\mathbf{K}^{1 / 2} \nabla \eta^{i+1}\right\|_{\Omega_{p}} \leq C \Delta t^{2} .
\end{aligned}
$$

We finish the proof.

\section{Numerical experiments}

In order to show the performance of our method, we give some numerical results in this section. Let $\Omega_{f}=[0,1] \times[1,2]$ and $\Omega_{p}=[0,1] \times[0,1]$ with interface $\Gamma=(0,1) \times\{1\}$. The exact solution is

$$
\begin{aligned}
& u_{1}(x, y, t)=\left[x^{2}(y-1)^{2}+y\right] \cos (t), \\
& u_{2}(x, y, t)=\left[-\frac{2}{3} x(y-1)^{3}\right] \cos (t)+[2-\pi \sin (\pi x)] \cos (t), \\
& p(x, y, t)=[2-\pi \sin (\pi x)] \sin (0.5 \pi y) \cos (t), \\
& \phi(x, y, t)=[2-\pi \sin (\pi x)][1-y-\cos (\pi y)] \cos (t) .
\end{aligned}
$$

The initial conditions, boundary conditions and the forcing terms are given by the exact solution. The finite element spaces choosing the MINI elements $(P 1 b-P 1)$ for the NavierStokes equation in $\Omega_{f}$ and the linear Lagrangian elements $(P 1)$ for the Darcy flow in $\Omega_{p}$. Here, we use the software package FreeFEM++ [28] for our program.

We introduce a more accurate approach $[36,38]$ to examine the orders of convergence with respect to the time step $\Delta t$ or the mesh size $h$ due to the approximation errors $\mathcal{O}\left(\Delta t^{r_{1}}\right)+\mathcal{O}\left(h^{r_{2}}\right)$. Then, we can assume that

$$
v_{h}^{\Delta t}\left(x, t_{m}\right) \approx v\left(x, t_{m}\right)+C_{1}\left(x, t_{m}\right) \Delta t^{r_{1}}+C_{2}\left(x, t_{m}\right) h^{r_{2}} .
$$

Thus, we can get

$$
\begin{aligned}
\rho_{v, h, i} & =\frac{\left\|v_{h}^{\Delta t}\left(x, t_{m}\right)-v_{h / 2}^{\Delta t}\left(x . t_{m}\right)\right\|_{i}}{\left\|v_{h / 2}^{\Delta t}\left(x, t_{m}\right)-v_{h / 4}^{\Delta t}\left(x . t_{m}\right)\right\|_{i}} \approx \frac{4^{r_{2}}-2^{r_{2}}}{2^{r_{2}}-1}, \\
\rho_{v, \Delta t, i} & =\frac{\left\|v_{h}^{\Delta t}\left(x, t_{m}\right)-v_{h}^{\Delta t / 2}\left(x . t_{m}\right)\right\|_{i}}{\left\|v_{h}^{\Delta t / 2}\left(x, t_{m}\right)-v_{h}^{\Delta t / 4}\left(x . t_{m}\right)\right\|_{i}} \approx \frac{4^{r_{1}}-2^{r_{1}}}{2^{r_{1}}-1} .
\end{aligned}
$$

Here, $v$ can be $u, p$ or $\phi$ and $i$ can be 0 or 1 . We can see that $\rho_{v, h, i}, \rho_{v, \Delta t, i}$ approach 4.0 or 2.0, the convergence order will be 2.0 or 1.0, respectively.

Firstly, we focus on the convergence orders with respect to the spacing step $h$, we study the errors with a fixed time step $\Delta t=0.01$ and varying spacing steps $h=$ $1 / 2,1 / 4,1 / 8,1 / 16$, and $1 / 32$, respectively. Tables 1,3 and 5 present the errors between 
Table 1: The numerical results at $T=1$ with $\Delta t=0.01, v=1.0$ and $\theta=0.05$ for different $h$.

\begin{tabular}{||c|c|c|c|c||}
\hline $1 / h$ & $\frac{\left\|u(T)-u_{h}^{N}\right\|_{0}}{\|u\|_{0}}$ & $\frac{\left\|\nabla\left(u(T)-u_{h}^{N}\right)\right\|_{0}}{\|u\|_{0}}$ & $\frac{\left\|\phi(T)-\phi_{h}^{N}\right\|_{0}}{\|\phi(T)\|_{0}}$ & $\frac{\left\|\nabla\left(\phi(T)-\phi_{h}^{N}\right)\right\|_{0}}{\|\nabla \phi(T)\|_{0}}$ \\
\hline 2 & 0.232865 & 0.401518 & 0.598926 & 0.628749 \\
4 & 0.0746141 & 0.210431 & 0.188994 & 0.35896 \\
8 & 0.0467415 & 0.117158 & 0.0471332 & 0.187352 \\
16 & 0.044809 & 0.077671 & 0.0114311 & 0.0950967 \\
32 & 0.0447816 & 0.064098 & 0.011852 & 0.0483665 \\
\hline
\end{tabular}

Table 2: Convergence order of $\mathcal{O}\left(h^{r}\right) T=1$ with $\Delta t=0.01, v=1.0$ and $\theta=0.05$ for different $h$.

\begin{tabular}{||c|c|c|c|c||}
\hline $1 / h$ & $\left\|u_{h}^{N}-u_{h / 2}^{N}\right\|_{0}$ & $\left\|\nabla\left(u_{h}^{N}-u_{h / 2}^{N}\right)\right\|_{0}$ & $\left\|\phi_{h}^{N}-\phi_{h / 2}^{N}\right\|_{0}$ & $\left\|\nabla\left(\phi_{h}^{N}-\phi_{h / 2}^{N}\right)\right\|_{0}$ \\
\hline 4 & 0.186536 & 1.36784 & 0.160306 & 1.40745 \\
8 & 0.0502415 & 0.710559 & 0.0519997 & 0.798709 \\
16 & 0.0126097 & 0.357623 & 0.0147521 & 0.416217 \\
32 & 0.00314492 & 0.178817 & 0.00382829 & 0.209643 \\
\hline & $\rho_{u, h, 0}$ & $\rho_{u, h, 1}$ & $\rho_{\phi, h, 0}$ & $\rho_{\phi, h, 1}$ \\
\hline 8 & 3.71279 & 1.92502 & 3.08283 & 1.76215 \\
16 & 3.98436 & 1.98689 & 3.5249 & 1.91898 \\
32 & 4.00954 & 1.99994 & 3.85344 & 1.98536 \\
\hline
\end{tabular}

Table 3: The numerical results at $T=1$ with $\Delta t=0.01, v=0.01$ and $\theta=4.0$ for different $h$.

\begin{tabular}{||c|c|c|c|c||}
\hline $1 / h$ & $\frac{\left\|u(T)-u_{h}^{N}\right\|_{0}}{\|u\|_{0}}$ & $\frac{\left\|\nabla\left(u(T)-u_{h}^{N}\right)\right\|_{0}}{\|u\|_{0}}$ & $\frac{\left\|\phi(T)-\phi_{h}^{\mathrm{N}}\right\|_{0}}{\|\phi(T)\|_{0}}$ & $\frac{\left\|\nabla\left(\phi(T)-\phi_{h}^{\mathrm{N}}\right)\right\|_{0}}{\|\nabla \phi(T)\|_{0}}$ \\
\hline 2 & 0.333976 & 0.831983 & 0.592141 & 0.629023 \\
4 & 0.0693539 & 0.431048 & 0.195919 & 0.358844 \\
8 & 0.0685211 & 0.252043 & 0.0542478 & 0.187652 \\
16 & 0.0775053 & 0.198443 & 0.0178825 & 0.0960285 \\
32 & 0.0808675 & 0.186711 & 0.0140446 & 0.0505207 \\
\hline
\end{tabular}

Table 4: Convergence order of $\mathcal{O}\left(h^{r}\right) T=1$ with $\Delta t=0.01, v=0.01$ and $\theta=4.0$ for different $h$.

\begin{tabular}{||c|c|c|c|c||}
\hline $1 / h$ & $\left\|u_{h}^{N}-u_{h / 2}^{N}\right\|_{0}$ & $\left\|\nabla\left(u_{h}^{N}-u_{h / 2}^{N}\right)\right\|_{0}$ & $\left\|\phi_{h}^{N}-\phi_{h / 2}^{N}\right\|_{0}$ & $\left\|\nabla\left(\phi_{h}^{N}-\phi_{h / 2}^{N}\right)\right\|_{0}$ \\
\hline 4 & 0.314933 & 3.25999 & 0.15549 & 1.40043 \\
8 & 0.0831566 & 1.64711 & 0.0518011 & 0.797107 \\
16 & 0.0200227 & 0.789583 & 0.014627 & 0.415866 \\
32 & 0.00534704 & 0.397218 & 0.00381673 & 0.209583 \\
\hline & $\rho_{u, h, 0}$ & $\rho_{u, h, 1}$ & $\rho_{\phi, h, 0}$ & $\rho_{\phi, h, 1}$ \\
\hline 8 & 3.78723 & 1.97922 & 3.00167 & 1.7569 \\
16 & 4.15311 & 2.08605 & 3.54148 & 1.91674 \\
32 & 3.74463 & 1.98778 & 3.83233 & 1.98425 \\
\hline
\end{tabular}

the numerical results and the exact solutions for $v=1.0,0.01$ and 0.001 , respectively. We can see that the errors diminish with the spacing step $h$ changing small. Tables 2,4 and 6 show the convergence orders with respect to the spacing step $h$ for $v=1.0,0.01$ and 0.001 , respectively. We can see that $\rho_{u, h, 0}$ and $\rho_{\phi, h, 0}$ are nearly 4.0 and $\rho_{u, h, 1}, \rho_{\phi, h, 1}$ approach 2.0. It suggests that the convergence order in space for the $L^{2}$-norm of $u_{h}$ and $\phi_{h}$ are $\mathcal{O}\left(h^{2}\right)$, 
Table 5: The numerical results at $T=1$ with $\Delta t=0.01, v=0.001$ and $\theta=4.0$ for different $h$.

\begin{tabular}{||c|c|c|c|c||}
\hline $1 / h$ & $\frac{\left\|u(T)-u_{h}^{N}\right\|_{0}}{\|u\|_{0}}$ & $\frac{\left\|\nabla\left(u(T)-u_{h}^{N}\right)\right\|_{0}}{\|u\|_{0}}$ & $\frac{\left\|\phi(T)-\phi_{h}^{N}\right\|_{0}}{\|\phi(T)\|_{0}}$ & $\frac{\left\|\nabla\left(\phi(T)-\phi_{h}^{N}\right)\right\|_{0}}{\|\nabla \phi(T)\|_{0}}$ \\
\hline 2 & 0.349949 & 0.925095 & 0.591913 & 0.629033 \\
4 & 0.0746775 & 0.500333 & 0.195544 & 0.358865 \\
8 & 0.0732168 & 0.291469 & 0.0536393 & 0.187717 \\
16 & 0.0820637 & 0.223925 & 0.0177584 & 0.0961669 \\
32 & 0.0854242 & 0.208026 & 0.0145202 & 0.0508133 \\
\hline
\end{tabular}

Table 6: Convergence order of $\mathcal{O}\left(h^{r}\right) T=1$ with $\Delta t=0.01, v=0.001$ and $\theta=4.0$ for different $h$.

\begin{tabular}{||c|c|c|c|c||}
\hline $1 / h$ & $\left\|u_{h}^{N}-u_{h / 2}^{N}\right\|_{0}$ & $\left\|\nabla\left(u_{h}^{N}-u_{h / 2}^{N}\right)\right\|_{0}$ & $\left\|\phi_{h}^{N}-\phi_{h / 2}^{N}\right\|_{0}$ & $\left\|\nabla\left(\phi_{h}^{N}-\phi_{h / 2}^{N}\right)\right\|_{0}$ \\
\hline 4 & 0.33257 & 3.64765 & 0.155497 & 1.40032 \\
8 & 0.0881533 & 1.89566 & 0.0519193 & 0.797389 \\
16 & 0.0213933 & 0.924649 & 0.0146362 & 0.416026 \\
32 & 0.0057549 & 0.467865 & 0.00381922 & 0.209667 \\
& $\rho_{u, h, 0}$ & $\rho_{u, h, 1}$ & $\rho_{\phi, h, 0}$ & $\rho_{\phi, h, 1}$ \\
8 & 3.77263 & 1.92421 & 2.99498 & 1.75613 \\
16 & 4.12061 & 2.05015 & 3.54733 & 1.91668 \\
32 & 3.7174 & 1.97632 & 3.83224 & 1.98422 \\
\hline
\end{tabular}

Table 7: The numerical results at $T=1$ with $h=1 / 32, v=1.0$ and $\theta=4.0$ for different $\Delta t$.

\begin{tabular}{||c|c|c|c|c||}
\hline$\Delta t$ & $\frac{\left\|u(T)-u_{h}^{N}\right\|_{0}}{\|u\|_{0}}$ & $\frac{\left\|\nabla\left(u(T)-u_{h}^{N}\right)\right\|_{0}}{\|u\|_{0}}$ & $\frac{\left\|\phi(T)-\phi_{h}^{N}\right\|_{0}}{\|\phi(T)\|_{0}}$ & $\frac{\left\|\nabla\left(\phi(T)-\phi_{h}^{N}\right)\right\|_{0}}{\|\nabla \phi(T)\|_{0}}$ \\
\hline 0.5 & 0.0600635 & 0.0684851 & 0.0231833 & 0.0493908 \\
0.25 & 0.027584 & 0.0476142 & 0.00652645 & 0.0477285 \\
0.125 & 0.0237243 & 0.0454769 & 0.00438339 & 0.0476608 \\
0.0625 & 0.0208114 & 0.0436585 & 0.00369908 & 0.0476051 \\
0.03125 & 0.0168278 & 0.0410535 & 0.00317619 & 0.0475391 \\
\hline
\end{tabular}

Table 8: Convergence order of $\mathcal{O}\left(\Delta t^{r}\right) T=1$ with $h=1 / 32, v=1.0$ and $\theta=4.0$ for different $\Delta t$.

\begin{tabular}{||c|c|c|c|c||}
\hline$\Delta t$ & $\left\|u_{h}^{N}-u_{h / 2}^{N}\right\|_{0}$ & $\left\|\nabla\left(u_{h}^{N}-u_{h / 2}^{N}\right)\right\|_{0}$ & $\left\|\phi_{h}^{N}-\phi_{h / 2}^{N}\right\|_{0}$ & $\left\|\nabla\left(\phi_{h}^{N}-\phi_{h / 2}^{N}\right)\right\|_{0}$ \\
\hline 0.25 & 0.178712 & 0.466978 & 0.0624593 & 0.465792 \\
0.125 & 0.0430235 & 0.126154 & 0.0156514 & 0.117084 \\
0.0625 & 0.00985868 & 0.0422007 & 0.00384549 & 0.0289859 \\
0.03125 & 0.0079011 & 0.036303 & 0.00106173 & 0.0082662 \\
\hline & $\rho_{u, \Delta t, 0}$ & $\rho_{u, \Delta t, 1}$ & $\rho_{\phi, \Delta t, 0}$ & $\rho_{\phi, \Delta t, 1}$ \\
\hline 0.125 & 4.15383 & 3.70166 & 3.99066 & 3.97827 \\
0.0625 & 4.36402 & 2.98937 & 4.07005 & 4.03934 \\
0.03125 & 1.24776 & 1.16246 & 3.6219 & 3.50656 \\
\hline
\end{tabular}

the convergence order in space for the $H^{1}$-norm of $u_{h}$ and $\phi_{h}$ are $\mathcal{O}\left(h^{1}\right)$.

Then, we focus on the convergence orders with respect to the time step $\Delta t$, we study the errors with a fixed time step $h=1 / 32$ and varying time steps $\Delta t=0.5,0.25,0.125,0.0625$ and 0.03125 , respectively. Tables 7,9 and 11 present the errors between the numerical results and the exact solutions for $v=1.0,0.01$ and 0.001 , respectively. We can see that the 
Table 9: The numerical results at $T=0.01$ with $h=1 / 32, v=0.01$ and $\theta=4.0$ for different $\Delta t$.

\begin{tabular}{||c|c|c|c|c||}
\hline$\Delta t$ & $\frac{\left\|u(T)-u_{h}^{N}\right\|_{0}}{\|u\|_{0}}$ & $\frac{\left\|\nabla\left(u(T)-u_{h}^{N}\right)\right\|_{0}}{\|u\|_{0}}$ & $\frac{\left\|\phi(T)-\phi_{h}^{N}\right\|_{0}}{\|\phi(T)\|_{0}}$ & $\frac{\left\|\nabla\left(\phi(T)-\phi_{h}^{N}\right)\right\|_{0}}{\|\nabla \phi(T)\|_{0}}$ \\
\hline 0.5 & 0.0815619 & 0.0991371 & 0.029804 & 0.0507619 \\
0.25 & 0.0293946 & 0.063132 & 0.0065347 & 0.0477487 \\
0.125 & 0.0237782 & 0.0579196 & 0.00417864 & 0.0476713 \\
0.0625 & 0.0203077 & 0.0537641 & 0.00353974 & 0.0476047 \\
0.03125 & 0.0160209 & 0.0492438 & 0.00306932 & 0.0475339 \\
\hline
\end{tabular}

Table 10: Convergence order of $\mathcal{O}\left(\Delta t^{r}\right) T=1$ with $h=1 / 32, v=1.0$ and $\theta=4.0$ for different $\Delta t$.

\begin{tabular}{||c|c|c|c|c||}
\hline$\Delta t$ & $\left\|u_{h}^{N}-u_{h / 2}^{N}\right\|_{0}$ & $\left\|\nabla\left(u_{h}^{N}-u_{h / 2}^{N}\right)\right\|_{0}$ & $\left\|\phi_{h}^{N}-\phi_{h / 2}^{N}\right\|_{0}$ & $\left\|\nabla\left(\phi_{h}^{N}-\phi_{h / 2}^{N}\right)\right\|_{0}$ \\
\hline 0.25 & 0.188199 & 0.414847 & 0.0645817 & 0.478491 \\
0.125 & 0.0444021 & 0.120286 & 0.0159055 & 0.118791 \\
0.0625 & 0.0109503 & 0.0573838 & 0.0037441 & 0.0284255 \\
0.03125 & 0.00857969 & 0.0583418 & 0.00103058 & 0.00794661 \\
\hline & $\rho_{u, \Delta t, 0}$ & $\rho_{u, \Delta t, 1}$ & $\rho_{\phi, \Delta t, 0}$ & $\rho_{\phi, \Delta t, 1}$ \\
\hline 0.125 & 4.23852 & 3.44883 & 4.06033 & 4.028 \\
0.0625 & 4.05487 & 2.09617 & 4.24816 & 4.17904 \\
0.03125 & 1.27631 & 0.983579 & 3.63302 & 3.57706 \\
\hline
\end{tabular}

Table 11: The numerical results at $T=0.01$ with $h=1 / 32, v=0.001$ and $\theta=4.0$ for different $\Delta t$.

\begin{tabular}{||c|c|c|c|c||}
\hline$\Delta t$ & $\frac{\left\|u(T)-u_{h}^{N}\right\|_{0}}{\|u\|_{0}}$ & $\frac{\left\|\nabla\left(u(T)-u_{h}^{N}\right)\right\|_{0}}{\|u\|_{0}}$ & $\frac{\left\|\phi(T)-\phi_{h}^{N}\right\|_{0}}{\|\phi(T)\|_{0}}$ & $\frac{\left\|\nabla\left(\phi(T)-\phi_{h}^{N}\right)\right\|_{0}}{\|\nabla \phi(T)\|_{0}}$ \\
\hline 0.5 & 0.0821624 & 0.0999722 & 0.0297175 & 0.0507326 \\
0.25 & 0.0295736 & 0.0636198 & 0.00653324 & 0.0477496 \\
0.125 & 0.0239263 & 0.0583415 & 0.00417921 & 0.0476718 \\
0.0625 & 0.0204167 & 0.0541207 & 0.00353977 & 0.047605 \\
0.03125 & 0.0160864 & 0.0495441 & 0.00306868 & 0.0475339 \\
\hline
\end{tabular}

Table 12: Convergence order of $\mathcal{O}\left(\Delta t^{r}\right) T=1$ with $h=1 / 32, v=0.001$ and $\theta=4.0$ for different $\Delta t$.

\begin{tabular}{||c|c|c|c|c||}
\hline$\Delta t$ & $\left\|u_{h}^{N}-u_{h / 2}^{N}\right\|_{0}$ & $\left\|\nabla\left(u_{h}^{N}-u_{h / 2}^{N}\right)\right\|_{0}$ & $\left\|\phi_{h}^{N}-\phi_{h / 2}^{N}\right\|_{0}$ & $\left\|\nabla\left(\phi_{h}^{N}-\phi_{h / 2}^{N}\right)\right\|_{0}$ \\
\hline 0.25 & 0.188326 & 0.413749 & 0.0645731 & 0.478325 \\
0.125 & 0.0444182 & 0.120054 & 0.0159067 & 0.118797 \\
0.0625 & 0.0110042 & 0.0578813 & 0.00374262 & 0.0284173 \\
0.03125 & 0.00866925 & 0.0590004 & 0.00103091 & 0.00794814 \\
\hline & $\rho_{u, \Delta t, 0}$ & $\rho_{u, \Delta t, 1}$ & $\rho_{\phi, \Delta t, 0}$ & $\rho_{\phi, \Delta t, 1}$ \\
\hline 0.125 & 4.23984 & 3.44635 & 4.05949 & 4.02642 \\
0.0625 & 4.03646 & 2.07414 & 4.25015 & 4.18043 \\
0.03125 & 1.26934 & 0.981033 & 3.63041 & 3.57533 \\
\hline
\end{tabular}

errors diminish with the time step $\Delta t$ changing small. Tables 8,10 and 12 show the convergence orders with respect to the time step $\Delta t$ for $v=1.0,0.01$ and 0.001 , respectively. We can see that $\rho_{u, \Delta t, 0}, \rho_{\phi, \Delta t, 0}, \rho_{u, \Delta t, 1}$ and $\rho_{\phi, \Delta t, 1}$ approach 4.0. It suggests that the convergence order in time for the $L^{2}$-norm of $u_{h}$ and $\phi_{h}$ and the $H^{1}$-norm of $u_{h}$ and $\phi_{h}$ are super convergence, which is an interesting thing. 


\section{Acknowledgements}

The authors would like to thank the anonymous referees for their valuable suggestions and comments, which helped to improve the quality of the paper.

\section{References}

[1] I. BABUŠKA AND G. N. GATICA, A residual-based a posteriori error estimator for the StokesDarcy coupled problem, SIAM J. Numer. Anal., 48(2) (2010), pp. 498-523.

[2] S. BADIA AND R. CODINA, Unified stabilized finite element formulations for the Stokes and the Darcy problems, SIAM J. Numer. Anal., 47(3) (2009), pp. 1971-2000.

[3] L. BAdeA, M. Discacciati AND A. QUARTERONI, Numerical analysis of the NavierStokes/Darcy coupling, Numer. Math., 115(2) (2010), pp. 195-227.

[4] G. S. BEAVERS AND D. D. JOSEPH, Boundary conditions at a naturally permeable wall, J. Fluid Mech., 30 (1967), pp. 197-207.

[5] J. BLASCO AND R. CODINA, Error estimates for an operator-splitting method for incompressible flows, Appl. Numer. Math., 51 (2004), pp. 1-17.

[6] J. BlASCO, R. CODINA AND A. HUERTA, A fractional-step method for the incompressible NavierStokes equations related to a predictor-multicorrector algorithm, Int. J. Numer. Methods Fluids, 28 (1998), pp. 1391-1419.

[7] M. C. CAI, M. MU AND J. C. XU, Numerical solution to a mixed Navier-Stokes/Darcy model by the two-grid approach, SIAM J. Numer. Anal., 47(5) (2009), pp. 3325-3338.

[8] J. Camaño, G. N. Gatica, R. Oyarzúa, R. Ruiz-Baier and P. Venegas, New fullymixed finite element methods for the Stokes-Darcy coupling, Comput. Method. Appl. Mech. Eng., 295 (2015), pp. 362-395.

[9] Y. Z. CaO, M. GunZBuRger, X. M. He AND X. M. WANG, Robin-Robin domain decomposition methods for the steady-state Stokes-Darcy system with the Beavers-Joseph interface condition, Numer. Math., 117(4) (2011), pp. 601-629.

[10] Y. Z. CaO, M. GunZburger, X. L. Hu, F. Hua, X. M. WANG AND W. D. ZhaO, Finite element approximations for Stokes-Darcy flow with Beavers-Joseph interface conditions, SIAM J. Numer. Anal., 47(6) (2010), pp. 4239-4256.

[11] A. J. CHORIN, Numerical solution of the Navier-Stokes equations, Math. Comput., 22 (1968), pp. $745-762$.

[12] W. B. Chen, M. Gunzburger, F. Hua And X. M. WANG, A parallel Robin-Robin domain decomposition method for the Stokes-Darcy system, SIAM J. Numer. Anal., 49(3) (2011), pp. 10641084.

[13] R. Codina, Pressure stability in fractional step finite element methods for incompressible flows, J. Comput. Phys., 170 (2001), pp. 112-140.

[14] R. CODINA AND S. BADIA, On some pressure segregation methods of fractional-step type for the finite element approximation of incompressible flow problems, Comput. Method Appl. Mech. Eng., 195 (2006), pp. 2900-2918.

[15] M. CUi AND N. N. YAN, A posteriori error estimate for the Stokes-Darcy system, Math. Method Appl. Sci., 34(9) (2011), pp. 1050-1064.

[16] M. DisCACCIATI AND A. QuARTERONI, Navier-Stokes/Darcy coupling: Modeling, analysis and numerical approximation, Rev. Mat. Complut., 22(2) (2009), pp. 315-426. 
[17] M. Discacciati, A. Quarteroni AND A. VAlli, Robin-Robin domain decomposition methods for the Stokes-Darcy coupling, SIAM J. Numer. Anal., 45(3) (2007), pp. 1246-1268.

[18] X. FENG, Y. HE AND P. HUANG, A stabilized implicit fractional-step method for the timedependent Navier-Stokes equations using equal-order pairs, J. Math. Anal. Appl., 392 (2012), pp. 209-224.

[19] X. FenG, Y. He AND D. LIU, Convergence analysis of an implicit fractional-step method for the incompressible Navier-Stokes equations, Appl. Math. Model, 35 (2011), pp. 5856-5871.

[20] E. FERNÁ-CARA AND M. MARÍN BELTRÁN, The convergence of two numerical schemes for the Navier-Stokes equations, Numer. Math., 55 (1989), pp. 33-60.

[21] V. GiRAult AND P. A. RAVIART, Finite Element Method for Navier-Stokes Equations: Theory and Algorithms, Springer-Verlag, Berlin, Herdelberg, 1987.

[22] F. GuilléN-GONZÁleZ AND M. V. REDONDO-Neble, New error estimates for a viscositysplitting scheme in time for the three-dimensional Navier-Stokes equations, IMA J. Numer. Anal., 31 (2011), pp. 556-579.

[23] G. N. Gatica, R. OYARzUA AND F. J. SAYAS, Analysis of fully-mixed finite element methods for the Stokes-Darcy coupled problem, Math. Comput., 80(276) (2011), pp. 1911-1948.

[24] G. N. Gatica, A. Márquez, R. Oyarzúa AND R. Rebolledo, Analysis of an augmented fully-mixed approach for the coupling of quasi-newtonian fluids and porous media, Comput. Method Appl. Mech. Eng., 270 (2014), pp. 76-112.

[25] V. GiRAult AND B. RiviÉRE, DG approximation of coupled Navier-Stokes and Darcy equations by Beaver-Joseph-Saffman interface condition, SIAM J. Numer. Anal., 47(3) (2009), pp. 20522089.

[26] F. Guillén-GonZÁlez AND M. V. RedOndo-Neble, New error estimates for a viscositysplitting scheme in time for the three-dimensional Navier-Stokes equations, IMA J. Numer. Anal., 31 (2011), pp. 556-579.

[27] R. Glowinski, T. W. PAn AND J. Periaux, A fictitious domain method for external incompressible viscous flow modeled by Navier-Stokes equations, Comput. Methods Appl. Mech. Eng., 112 (1994), pp. 133-148.

[28] F. Hecht, New development in FreeFem++, J. Numer. Math., 20 (2012), pp. 251-265.

[29] Y. Hou, Optimal error estimates of a decoupled scheme based on two-grid finite element for mixed Stokes-Darcy model, Appl. Math. Lett., 57 (2016), pp. 90-96

[30] G. KANSCHAT AND B. RIVIERE, A strongly conservative finite element method for the coupling of Stokes and Darcy flow, J. Comput. Phys., 29(17) (2010), pp. 5933-5943.

[31] W. L. LAYTON, H. TRAN AND X. XIONG, Long time stability of four methods for splitting the evolutionary Stokes-Darcy problems into Stokes and Darcy subproblems, J. Comput. Appl. Math., 236(13) (2012), pp. 3198-3217.

[32] A. MÁrquez, S. MedDAhi AND F.-J. SAYAS, A decoupled preconditioning technique for a mixed Stokes-Darcy model, J. Sci. Comput., 57 (2013), pp. 174-192.

[33] A. MÁRQUeZ, S. MEdDAHI AND F.-J. SAYAS, Strong coupling of finite element methods for the Stokes-Darcy problem, IMA J. Numer. Anal., 35(2) (2015), pp. 969-988.

[34] M. MORAITI, On the quasistatic approximation in the Stokes-Darcy model of groundwater-surface water flows, J. Math. Anal. Appl., 394 (2012), pp. 796-808.

[35] M. MU AND J. C. XU, A two-grid method of a mixed Stokes-Darcy model for coupling fluid flow with porous media flow, SIAM J. Numer. Anal., 45(5) (2007), pp. 1801-1813.

[36] M. MU AND X. H. ZHU, Decoupled schemes for a non-stationary mixed Stokes-Darcy model, Math. Comput., 79(270) (2010), pp. 707-731.

[37] P. G. SAFFMAN, On the boundary at the surface of a porous medium, Studies Appl. Math., 50 
(1971), pp. 93-101.

[38] L. ShAN, H. ZHeng AND W. J. LAYTON, A decoupling method with different subdomain time steps for the nonstationary Stokes-Darcy model, Numer. Methods Partial Differential Equations, 29 (2013), pp. 549-583.

[39] Z. SI, Y. WANG AND S. LI, Decoupled modified characteristics finite element method for the time dependent Navier-Stokes/Darcy problem, Math. Method Appl. Sci., 37 (2014), pp. 1392-1404.

[40] R. Temam, Une méthode d'approximations de la solution des equations de Navier-Stokes, Bull. Soc. Math. Fr., 98 (1968), pp. 115-152.

[41] D. VASSILEV AND I. YOTOV, Coupling Stokes-Darcy flow with transport, SIAM J. Sci. Comput., 31(5) (2009), pp. 3661-3684.

[42] T. Zhang, D. Pedro AND J. YUAN, A large time stepping viscosity-splitting finite element method for the viscoelastic flow problem, Adv. Comput. Math., 41 (2015), pp. 149-190.

[43] T. ZHANG AND J. Y. YUAN, Two novel decoupling algorithms for the steady Stokes-Darcy model based on two-grid discretizations, Discrete Cont. Dyn. B, 19 (2014), pp. 849-865.

[44] L. ZUO AND Y. HOU, A decoupling two-grid algorithm for the mixed Stokes-Darcy model with the Beavers-Joseph interface condition, Numer. Methods Partial Differential Equations, 30(3) (2014), pp. 1066-1082 